\title{
A Class of Homogeneous Cosmological Models
}

\author{
G. F. R. Ellis and M. A. H. MacCallum \\ Department of Applied Mathematics and Theoretical Physics, \\ Silver Street, Cambridge, England \\ Received November 11, 1968
}

\begin{abstract}
Einstein's field equations are studied under the assumptions that (1) the source of the gravitational field is a perfect fluid, and (2) there exists a group of motions simply transitive on three-surfaces orthogonal to the fluid flow vector. There are two classes of solutions; these are studied in detail. Three special families of solutions examined include all analytic solutions of the field equations obeying (1) and (2) of which the authors are aware. The relation of these solutions to various vacuum solutions is indicated.
\end{abstract}

\section{Introduction}

We shall consider solutions of Einstein's field equations

$$
R_{a b}-\frac{1}{2} R g_{a b}+\Lambda g_{a b}=T_{a b}
$$

in which the matter tensor takes the form of a perfect fluid

$$
T_{a b}=\mu u_{a} u_{b}+p\left(g_{a b}+u_{a} u_{b}\right), \quad u_{a} u^{a}=-1
$$

where $u^{a}$ is the normalised four-velocity, $\mu$ the density and $p$ the pressure of the fluid. We shall normally assume $\mu+p>0$. The Eqs. (1.1) and (1.2) are integrable provided we are given an equation of state. This we will usually assume to have the form $p=p(\mu)$. (Because of the homogeneity, this will be no restriction on our models unless $\mu$ takes the same value twice.)

Exact analytic solutions of these equations have, of necessity, high symmetry. The conservation equation $u_{a} T_{; b}^{a b}=0$ takes the form

$$
\mu^{\prime}:=\mu_{, a} u^{a}=-(\mu+p) \theta
$$

where $\theta:=u_{; a}^{a}$ is the expansion of the fluid (see Refs. $[1,3]$ for standard notation and results). Thus homogeneity of space-time, which implies a constant density $\mu$, also implies that $\theta=0$ and so we would not see an almost isotropic redshift. Hence such spacetimes are not reasonable cosmological models. The two simplest classes of spacetime that will give reasonable cosmological models are the well-known Friedmann universes, which are isotropic and are homogeneous on spacelike sections, and those 
spaces admitting a group of motions ${ }^{1} G_{4}$ multiply transitive on surfaces of homogeneity $S_{3}$. These spaces ${ }^{2}$ are included in the spacetimes classified and examined in two previous papers $[1,2]$.

We shall examine here the next simplest class of spacetimes suitable for use as cosmological models; that is, we assume that (1.1) and (1.2) hold, $\theta>0$ and that

there exists a group $G_{3}$ of motions simply transitive on three-surfaces orthogonal to the fluid flow vector $u^{a}$.

In these spacetimes matter expands and the universe is homogeneous in the rest-space of any fundamental observer. These are a subclass of the Bianchi spacetimes which were systematically investigated by TAUB [5] in the case $p=\mu=0$ and by ScHÜckING [6] in the case $p=0 \neq \mu$. The specialisation is that the fluid flow vector is orthogonal to the surfaces of transitivity of the group $G_{3}$; it is for this reason that the rest-spaces of the fundamental observers coincide with the surfaces of homogeneity (which is not the case, for example, in GöDEL's spacetimes [7]).

Since $u^{a}$ is hypersurface orthogonal, the vorticity tensor $\omega_{a b}$ is zero, and the acceleration vector $\dot{u}_{a}$ vanishes because the acceleration of the vector field normal to a family of spacelike hypersurfaces of transitivity of a group of motions is always zero [4]; that is.

$$
\begin{aligned}
\dot{u}_{a} & :=u_{a ; b} u^{b}=0, \\
\omega_{a b}: & =u_{[a ; b]}-\dot{u}_{[a} u_{b]}=0 .
\end{aligned}
$$

(1.5a) also follows from the conservation equations $\left(g_{a b}+u_{a} u_{b}\right) T_{; c}^{a c}=0$, which are

$$
(\mu+p) \dot{u}_{a}=-\left(g_{a}^{b}+u_{a} u^{b}\right) p, b
$$

since $p$ must be constant on the surfaces of transitivity of the group.

The Eqs. (1.5) imply that we may choose a time coordinate $t$ so that $\{t=$ constant $\}$ are the surfaces of transitivity of the group and $t,{ }_{a} u^{a}=1$ (i.e. $u_{a}=-t,,_{a}$ ) [3]. Since

$$
u_{a ; b}=\theta_{a b} \quad \text { where } \theta_{a b}=\theta_{(a b)} ; \theta_{a b} u^{b}=0
$$

the expansion tensor $\theta_{a b}$ completely determines the first derivative of $u_{a}$ and the first order redshift in the model. $\theta_{a b}$ may be split into the expansion $\theta=\theta_{a}^{a}$ and the symmetric trace-free tensor $\sigma_{a b}=\theta_{a b}$ $-\frac{1}{3} \theta\left(g_{a b}+u_{a} u_{b}\right)$, which is orthogonal to $u^{a}$. The Friedmann world models occur when $\sigma_{a b}=0$, or equivalently when $\sigma^{2}:=\frac{1}{2} \sigma_{a b} \sigma^{a b}$ vanishes.

${ }^{1}$ For terminology and results in the theory of groups of motions see (e.g.) Ref. [4].

${ }^{2}$ We shall call these spacetimes L.R.S. (locally rotationally symmetric). 
The group of motions $G_{3}$ can be classified into one of the nine distinct types described by Branchi [5, 8]. Since the group of motions is a Lie group and hence analytic, this Bianchi type is constant throughout connected non-singular regions of spacetime where the group acts regularly ${ }^{3}$. Although the Bianchi type is a useful description of the symmetry of spacetime, it does not describe the relation of the group to the metric tensor. In this paper we shall take into account both the group type and its relation to the metric tensor, using the group classification techniques developed by ScHÜCKING, KUNDT and BEHR, and the tetrad methods of Refs. [1] and [2]. Thus we use an orthonormal tetrad (used previously to study Bianchi world models in Refs. [9-11]) rather than one in which the scalar products of the tetrad vectors vary from surface to surface (used previously to study Bianchi world models in Refs. $[5,6,12-15])$.

Notation is very similar to that in $[1]: a, b, c, \ldots$ run from 0 to 3 ; $\alpha, \beta, \ldots$ from 1 to $3 ; \eta^{a b c d}=\eta^{[a b c d]}$ is a skew pseudo-tensor with tetrad components $\eta^{0123}=1 ; \varepsilon^{\alpha \beta \gamma}=\varepsilon^{[\alpha \beta \gamma]}$ is a skew pseudo-tensor with tetrad components $\varepsilon^{123}=1$. For arbitrary vectors $\boldsymbol{X}=X^{i} \frac{\partial}{\partial x^{i}}$, $\boldsymbol{Y}=Y^{i} \frac{\partial}{\partial x^{i}}$ the commutator $[\boldsymbol{X}, \boldsymbol{Y}]$ is the vector defined by

$$
[\boldsymbol{X}, \boldsymbol{Y}] f:=\boldsymbol{X}(\boldsymbol{Y} f)-\boldsymbol{Y}(\boldsymbol{X} f) \text { for all functions } f .
$$

With this definition, arbitrary vectors satisfy the Jacobi identity

$$
[\boldsymbol{X},[\boldsymbol{Y}, \boldsymbol{Z}]]+[\boldsymbol{Y},[\boldsymbol{Z}, \boldsymbol{X}]]+[\boldsymbol{Z},[\boldsymbol{X}, \boldsymbol{Y}]]=0 .
$$

Section 2 sets up the tetrad system, classifies the groups and gives the Jacobi equations, and Section 3 gives the field equations. Sections 4 and 5 proceed with integration. Sections 6,7 and 8 examine special cases of the group or metric structure. The results in Sections 4 to 8 are, as far as we are aware, new, unless otherwise indicated.

In the following sections we consider only local results. Since any world-model with homogeneous space sections becomes singular if the "energy condition" $\mu+3 p>0$ is satisfied [16], the local methods of integration used will break down if we consider sufficiently long times ${ }^{4}$.

\section{The Tetrad System}

We introduce as a vector basis an orthonormal tetrad $\left\{\boldsymbol{e}_{a}\right\}$, with the timelike vector $\boldsymbol{e}_{0}$ chosen as

$$
e_{0}^{j}=u^{j} .
$$

${ }^{3}$ There is no difficulty in extending a solution satisfying (1.4) locally to a global solution.

${ }^{4}$ In our models this follows directly from RAYCHAUDHURI's work [3, 17$]$. 
Then at each point the vectors $\left\{\boldsymbol{e}_{\gamma}\right\}$ form a triad of orthonormal spacelike vectors spanning the tangent plane to the surfaces of transitivity of the group; so the coordinate $x^{0}=t$ satisfies $t,{ }_{j} e_{\gamma}^{j}=0$. The other coordinates are chosen to be co-moving: $x^{\gamma}{ }_{j} u^{j}=0$. We use to denote covariant differentiation in the direction of the vector $\boldsymbol{u}$.

As the $\left\{\boldsymbol{e}_{a}\right\}$ are orthonormal, the metric tensor has the tetrad components

$$
g_{a b}:=e_{a}^{i} e_{b i}=\operatorname{diag}(-1,+1,+1,+1) .
$$

If $f$ is any function, the derivative of $f$ in the direction $\boldsymbol{e}_{a}$ is $\partial_{a} f:=f{ }_{i} e_{a}^{i}$. These derivatives do not commute in general and we define the functions $\gamma_{a b}^{c}$ by

$$
\left[\boldsymbol{e}_{a}, \boldsymbol{e}_{b}\right] f:=\left(\partial_{a} \partial_{b}-\partial_{b} \partial_{a}\right) f=: \gamma_{a b}^{c} \partial_{c} f, \gamma_{a b}^{c}=\gamma_{[a b]}^{c} .
$$

The rotation coefficients $\Gamma_{c a b}$ are defined by

$$
\Gamma_{\mathrm{\imath} a b}:=e_{c}^{j} e_{b j ; i} e_{a}^{i}
$$

so $g_{i j ; k}=0$ implies $\Gamma_{c a b}+\Gamma_{b a c}=0$.

Lowering and raising tetrad indices by $g_{a d}$ and its inverse $g^{a d}$ (note that $\left.g_{a d} \stackrel{*}{=} g^{a} a\right)$, it follows that

$$
\gamma_{a b}^{c}=\Gamma_{a b}^{c}-\Gamma_{b a}^{c} \Leftrightarrow \Gamma_{a b c}=\frac{1}{2}\left(\gamma_{a b c}+\gamma_{c a b}-\gamma_{b c a}\right) .
$$

From (2.1), (2.5) and (1.5)

$$
\gamma_{0 \gamma}^{0}=\gamma_{\mu \gamma}^{0}=0 .
$$

The Fermi-derivatives $\boldsymbol{e}_{\mu} \cdot \dot{\boldsymbol{e}}_{\nu}:=e_{\mu}^{i} e_{v i ; j} u^{j}$ may bo replaced, since $\boldsymbol{e}_{\mu} \cdot \dot{\boldsymbol{e}}_{v}=-\boldsymbol{e}_{v} \cdot \dot{\boldsymbol{e}}_{\mu}$, by the vector [18]

$$
\Omega^{a}:=\frac{1}{2} \eta^{a b c d} u_{b} \boldsymbol{e}_{c} \cdot \dot{\boldsymbol{e}}_{d}
$$

which implies $\Omega^{a} u_{a}=0 . \Omega^{a}$ is the local angular velocity, in the restframe of an observer with four-velocity $u^{a}$, of a set of Fermi-propagated axes with respect to the triad $\left\{\boldsymbol{e}_{v}\right\}$. Using (1.6) we have

$$
\gamma_{0 \nu}^{\mu}=-\theta_{\mu \nu}+\varepsilon_{\mu \nu \tau} \Omega^{\tau} .
$$

To describe the functions $\gamma_{\nu}^{\mu}$ we follow Schücking, Kundt and BEHR [19] in separating $\varepsilon^{\nu \sigma \tau} \gamma_{\nu \sigma}^{\mu}$ into a symmetric part $n^{\mu \tau}$ and an antisymmetric part represented by the "vector" $a^{\beta}$. These are defined by

$$
n^{\varkappa \tau}:=\frac{1}{2} \gamma_{\nu \sigma}^{(\kappa} \varepsilon^{\tau) v \sigma} ; a_{\beta}=\frac{1}{2} \gamma_{\beta \alpha}^{\alpha}
$$

the inverse of these relations being

$$
\gamma_{\mu \nu}^{\varkappa}=\varepsilon_{\mu \nu \tau} n^{\mu \tau}+\delta_{\nu}^{\varkappa} a_{\mu}-\delta_{\mu}^{\varkappa} a_{\nu} .
$$

The quantities $\theta_{\alpha \beta}, n_{\alpha \beta}, a_{\beta}, \Omega_{\beta}$, which completely determine the rotation coefficients $\Gamma_{a b c}$ (and $\gamma_{b c}^{a}$ ), behave as symmetric three-tensors and 
three-vectors under orthogonal transformations of the triad which are constant in each surface of transitivity.

The triad must lie in the three-surface of transitivity of the group of motions at each point, and we may further restrict it by

$$
\left[\boldsymbol{e}_{v}, \boldsymbol{\xi}_{\mu}\right]=0
$$

where $\boldsymbol{\xi}_{\mu}$ denotes a basis of the Killing vectors generating the simplytransitive $G_{3}$. A proof of this is to consider taking an orthonormal triad at one point $p$ in a surface $\{t=$ constant $\}$ and then dragging it along successively by $\xi_{1}$ to define it on a line, by $\boldsymbol{\xi}_{2}$ to define it on a twosurface, and finally by $\boldsymbol{\xi}_{3}$ to cover the hyperplane. Dragging along by a Killing vector preserves scalar products and hence the triad thus defined is orthonormal everywhere in the surface. The Jacobi identities now show ${ }^{5}$ that (2.6) holds and incidentally prove that the construction is independent of the choice of Killing vector basis. When the full group of motions is simply-transitive, an alternative proof of (2.6) can be given. Any invariant vector uniquely defined by the curvature tensor (or its covariant derivatives) commutes with the Killing vectors. As the group of motions is simply transitive (rather than multiply-transitive) we can find a covariantly and uniquely defined triad, so (2.6) holds for this triad. Further, since $\boldsymbol{u}$ is uniquely defined in all cases, $\left[\boldsymbol{\xi}_{v}, \boldsymbol{u}\right]=0$, and hence

$$
\left[\boldsymbol{\xi}_{v}, \boldsymbol{e}_{a}\right]=0 .
$$

These equations are entirely equivalent to Killing's equations $\xi_{v(a ; b)}=0$ for the vectors $\xi_{\nu}$.

We define $C_{\mu \nu}^{x}$ by

$$
\left[\boldsymbol{\xi}_{\mu}, \boldsymbol{\xi}_{\nu}\right]=C_{\mu \nu}^{\varkappa} \boldsymbol{\xi}_{\varkappa} ; C_{\mu \nu}^{\varkappa}=C_{[\mu \nu]}^{\varkappa} .
$$

Using (2.7) and applying the Jacobi identities to $\left(\boldsymbol{e}_{u}, \boldsymbol{\xi}_{\nu}, \boldsymbol{\xi}_{\mu}\right)$ we verify that the structure constants of the group, $C_{\mu \nu}^{*}$, are constant throughout spacetime [10] (cf. comment in Section 1). (2.7) and the Jacobi identities for $\left(\boldsymbol{\xi}_{l}, \boldsymbol{e}_{a}, \boldsymbol{e}_{b}\right)$ show that $\gamma_{b c}^{a}$ are constant in the surfaces of transitivity, i.e.

$$
\gamma_{b c}^{a}=\gamma_{b c}^{a}(t) .
$$

The vectors $\left\{\boldsymbol{e}_{v}\right\}$ thus generate a simply-transitive group of transformations in each surface $\{t=$ constant $\}$. This is the group reciprocal to the group of motions. Conversely, the existence of the group of transformations generated by $\left\{\boldsymbol{e}_{\nu}\right\}$ with the condition (2.8a) implies the existence of three independent $\boldsymbol{\xi}_{\mu}$ satisfying (2.6). The uniqueness theorem for solutions of differential equations then shows that (2.7) is satisfied,

5 This is essentially what is done in EISENHART's book (Ref. [3], p. 113); note that (2.8) is the statement that each isometry induces an automorphism of the Lie algebra. 
provided that

$$
\gamma_{0 v}^{0}=\gamma_{\mu v}^{0}=0
$$

Lemma 2.1. (1.4) holds if and only if there exists an orthonormal tetrad $\left\{e_{a}\right\}$ with $e_{0}=u$ such that (2.8) holds. The $\left\{e_{v}\right\}$ span the surfaces of transitivity of the group.

(2.8) shows that

$$
\begin{array}{ll}
\partial_{\gamma} \theta_{\alpha \beta}=0, & \partial_{\nu} \Omega_{\mu}=0, \\
\partial_{\gamma} n_{\alpha \beta}=0, & \partial_{\nu} a_{\mu}=0 .
\end{array}
$$

The Jacobi identities for $\left(\boldsymbol{e}_{a}, \boldsymbol{e}_{b}, \boldsymbol{e}_{c}\right)$ are, using (2.3),

$$
0=\partial_{a} \gamma_{b c}^{d}+\partial_{c} \gamma_{a b}^{d}+\partial_{b} \gamma_{c a}^{d}+\gamma_{a f}^{d} \gamma_{b c}^{f}+\gamma_{c f}^{d} \gamma_{a b}^{f}+\gamma_{b f}^{d} \gamma_{c a}^{f},
$$

which imply also the contracted identities

$$
0=\partial_{a} \gamma_{b c}^{a}+\partial_{c} \gamma_{a b}^{a}-\partial_{b} \gamma_{a c}^{a}+\gamma_{a f}^{a} \gamma_{b c}^{f} .
$$

Applying these relations to $\left(\boldsymbol{e}_{0}, \boldsymbol{e}_{\mu}, \boldsymbol{e}_{v}\right)$ shows that

$$
\partial_{0} a_{\alpha}+\sigma_{\alpha \beta} a^{\beta}+\frac{1}{3} \theta a_{\alpha}+\varepsilon_{\alpha \beta \gamma} \alpha^{\beta} \Omega^{\gamma}=0
$$

and

$$
\partial_{0} n_{\alpha \beta}+2 n^{\gamma}{ }_{(\alpha} \varepsilon_{\beta) \gamma \pi} \Omega^{\pi}-2 n_{\gamma(\alpha} \sigma_{\beta)}{ }^{\gamma}+\frac{1}{3} n_{\alpha \beta} \theta=0
$$

give the time derivatives of $a_{\alpha}, n_{\alpha \beta}$. Applying the identities to the vectors $\left(\boldsymbol{e}_{\mu}, \boldsymbol{e}_{\sigma}, \boldsymbol{e}_{v}\right)$ we see that for these vectors $(2.10 \mathrm{a}) \Leftrightarrow(2.10 \mathrm{~b})$ as each set contains only three independent relations, the identities $\left(\begin{array}{c}x \\ \mu \sigma \nu\end{array}\right)$ of Ref. [1]. By $(2.9 \mathrm{~b})$, these are

$$
n_{\alpha \beta} a^{\beta}=0 .
$$

As we are still permitted an arbitrary time-dependent rotation of the triad, we may make a canonical choice by taking the vectors $\left\{\boldsymbol{e}_{\nu}\right\}$ as eigenvectors of the symmetric "three-tensor" $n_{\alpha \beta}$. This assures orthogonality unless two eigenvalues are equal, in which case the vectors may be chosen to be orthogonal. As (2.13) shows $\alpha^{\beta}$ to be an eigenvector, we may take

$$
n_{\alpha \beta} \stackrel{*}{=} \operatorname{diag}\left(n_{1}, n_{2}, n_{3}\right), \quad a^{\beta} \stackrel{*}{=}(a, 0,0)
$$

without loss of generality, and then (2.13) reads

$$
a n_{1}=0
$$

and the commutators of the basis vectors $\left\{\boldsymbol{e}_{\boldsymbol{v}}\right\}$ are

$$
\left.\begin{array}{l}
{\left[\boldsymbol{e}_{1}, \boldsymbol{e}_{2}\right]=\alpha \boldsymbol{e}_{2}+n_{3} \boldsymbol{e}_{3},} \\
{\left[\boldsymbol{e}_{2}, \boldsymbol{e}_{3}\right]=n_{1} \boldsymbol{e}_{1},} \\
{\left[\boldsymbol{e}_{3}, \boldsymbol{e}_{1}\right]=n_{2} \boldsymbol{e}_{2}-a \boldsymbol{e}_{3} .}
\end{array}\right\}
$$


The three directions thus chosen are unique unless some of the eigenvalues of $n_{\alpha \beta}$ are equal, in which case there is a corresponding freedom of rotation, providing $(2.8)$ is preserved. Finally we have the freedom to reverse the direction of, and to renumber, the vectors. Using this freedom we may make $a$ positive if it is not zero, and simultaneously change the sign of all the $n_{\gamma}$. Thus we may bring the coefficients $\gamma_{v \sigma}^{\prime \prime}$ to one of the canonical forms in Table 1.

Table 1. Classification of groups $G_{3}$ following BeHR [10]. The group is of the type listed on the left, if it is possible to choose a basis such that the $\gamma_{\mu \nu}^{*}$ satisfy (2.8) and have the form on the right. The Jacobi identities (2.13) are identically satisfied by all these group types

\begin{tabular}{|c|c|c|c|c|c|c|}
\hline Group class & Group type & $a$ & $n_{1}$ & $n_{2}$ & $n_{3}$ & Bianchi type \\
\hline \multirow[t]{6}{*}{ A } & $I$ & 0 & 0 & 0 & 0 & I \\
\hline & II & 0 & + & 0 & 0 & II \\
\hline & $\mathrm{VII}_{0}$ & 0 & + & + & 0 & VII \\
\hline & $\mathrm{VI}_{0}$ & 0 & + & - & 0 & VI \\
\hline & IX & 0 & + & + & + & IX \\
\hline & VIII & 0 & + & + & - & VIII \\
\hline \multirow[t]{4}{*}{$\mathrm{B}$} & $\mathrm{V}$ & + & 0 & 0 & 0 & $\mathrm{~V}$ \\
\hline & IV & + & 0 & 0 & + & IV \\
\hline & $\mathrm{VII}_{h}$ & + & 0 & + & + & VII \\
\hline & $\mathrm{VI}_{h}$ & + & 0 & + & - & VI $\quad($ III if $h=-1)$ \\
\hline
\end{tabular}

Defining functions $\psi_{v}^{\mu}$ by $\boldsymbol{e}_{v}=\psi_{v}^{\mu} \boldsymbol{\xi}_{\mu},(2.6)$ and (2.8) imply [4]

$$
\psi_{\nu}^{\tau} \psi_{\mu}^{\alpha} C_{\tau \alpha}^{e}=\gamma_{\mu \nu}^{\alpha} \psi_{\kappa}^{e} .
$$

If we choose the Killing vector basis so that at one chosen point

$$
\psi_{\mu}^{\nu} \stackrel{*}{=} \delta_{\mu}^{\nu}
$$

then at that point $C_{\tau \alpha}^{o} \stackrel{*}{=} \gamma_{\tau \alpha}^{o}$ and hence the group of motions and its reciprocal group in each surface of transitivity are equivalent. At the point where (2.17) holds the Killing vector commutators have the form (2.16), but we may scale the basis arbitrarily and so we can make all non-zero structure constants either +1 or -1 , unless $a n_{2} n_{3} \neq 0$, in which case we may set $n_{2}, n_{3}$ to $\pm 1, a$ then being determined by the invariance of

$$
h:=a^{2} / n_{2} n_{3} .
$$

Thus there are 10 different types of group $G_{3}$ at a point, and since the $C_{\mu \nu}^{\varkappa}$ are constant there are 10 different types of group acting on space time. (Note that $h$ is time-invariant and that the values of $h$ in 
types $\mathrm{VII}_{h}$ and $\mathrm{VI}_{h}$ actually label one-parameter families of groups.) The preservation in time of group type may also be seen from (2.11) and (2.12). Table 1 gives the classification of $\mathrm{BEHR}$, and relates it to that of Bianchi (see Ref. [10]).

We shall refer to a group in which $a=0$ (i.e. in which $C_{\beta \alpha}^{\alpha}=0$ ), or a spacetime invariant under such a group and satisfying (1.1), (1.2) and (1.4) as of Class $A$, and a group in which $\alpha \neq 0$ (i.e. in which $C_{\beta \alpha}^{\alpha} \neq 0$ ), or a spacetime satisfying (1.1), (1.2) and (1.4), invariant under such a group, as being of Class $B$.

Since $n_{\alpha \beta}$ is symmetric there is a second canonical choice when $n:=n_{\alpha}^{\alpha}=0$. From Table $I$ this happens in types $I$ and $V$, and can happen in special cases of type $\mathrm{VI}_{0}, \mathrm{VI}_{h}$, and VIII. Then we may take an orthonormal triad $\left\{\boldsymbol{e}_{v}\right\}$ such that

$$
n_{\alpha \beta} \stackrel{*}{=}\left(\begin{array}{lll}
0 & r & r \\
r & 0 & q \\
r & q & 0
\end{array}\right) ; a_{\beta} \stackrel{*}{=}(a, 0,0) ; \quad a r=0 .
$$

The directions of the axes are determined by this form, unless $r=q$ in which case there is a rotation preserving (2.19). The commutators of the vectors $\left\{\boldsymbol{e}_{\gamma}\right\}$ are

$$
\left.\begin{array}{l}
{\left[\boldsymbol{e}_{1}, \boldsymbol{e}_{2}\right]=r \boldsymbol{e}_{1}+(q+a) \boldsymbol{e}_{2},} \\
{\left[\boldsymbol{e}_{2}, \boldsymbol{e}_{3}\right]=r \boldsymbol{e}_{2}+r \boldsymbol{e}_{3},} \\
{\left[\boldsymbol{e}_{3}, \boldsymbol{e}_{1}\right]=r \boldsymbol{e}_{1}+(q-a) \boldsymbol{e}_{3} .}
\end{array}\right\}
$$

This gives rise to the canonical forms of Table 2 .

Table 2. Classification of groups where $n_{\beta}^{\beta}=0$ (see Table 1). The Jacobi identities (2.19) are identically satisfied by these group types (see also Section 6)

\begin{tabular}{lllll}
\hline Group class & Group type & $a$ & $r$ & $q$ \\
\hline \multirow{2}{*}{$\mathrm{A}$} & $\mathrm{I}$ & 0 & 0 & 0 \\
& $\mathrm{VI}_{0}$ & 0 & 0 & + \\
& VIII & 0 & + & + \\
& & & & \\
$\mathrm{V}$ & $\mathrm{V}$ & + & 0 & 0 \\
& $\mathrm{VI}_{h}$ & + & 0 & + \\
\hline
\end{tabular}

The non-trivial Jacobi Eqs. (2.11), (2.12) are written out in Appendix I with the tetrad choice (2.14), and for the case $n_{\alpha}^{\alpha}=0$ in Appendix II with the tetrad choice (2.19). 


\section{The Field Equations}

From the Ricci identity for an arbitrary vector $v^{e}$,

$$
v_{; c d}^{b}-v_{; d c}^{b}=-R_{e c d}^{b} v^{e},
$$

we can find the Riemann tensor components and so the expression

$$
-R_{b d}:=-R_{b a d}^{a}=\partial_{d} \Gamma_{c b}^{c}-\partial_{c} \Gamma_{d b}^{c}-\Gamma_{c s}^{c} \Gamma_{d b}^{s}+\Gamma_{c b}^{s} \Gamma_{s d}^{c}
$$

for the Ricci tensor. Using (2.5) and the tetrad specialisations of the previous section, the field Eqs. (1.1), (1.2) are as follows.

$$
\theta+\frac{1}{3} \theta^{2}+2 \sigma^{2}+\frac{1}{2} \mu+\frac{3}{2} p=\Lambda
$$

is the $\left(\begin{array}{ll}0 & 0\end{array}\right)$ equation, Raychaudhuri's equation $[3,17]$, which shows that $\{\mu+3 p>0, \Lambda \leqq 0\} \Rightarrow\left\{\theta^{\circ}<0\right.$ at all times $\}$;

$$
3 a_{\varkappa} \sigma_{v}^{\varkappa}-\varepsilon_{\nu \varkappa \tau} n^{\tau \mu} \sigma_{\mu}^{\varkappa}=0
$$

is the $(0 v)$ equation stating that $u^{a}$ is a Ricci eigenvector; and, using (3.2), we can write the trace-free part of the $(\beta \delta)$ equations as

$$
\begin{aligned}
\partial_{0} \sigma_{\beta \delta}=-\theta \sigma_{\beta \delta}+2 \sigma^{\varkappa}{ }_{(\beta} \varepsilon_{\delta) \tau \varkappa} \Omega^{\tau} & +2 \varepsilon_{\tau \sigma(\beta} n_{\delta)}{ }^{\tau} \alpha^{\sigma}-2 n_{\delta_{\mu}} n_{\beta}^{\mu}+n n_{\beta \delta} \\
& +\frac{1}{3} \delta_{\beta \delta}\left(2 n^{\varkappa \tau} n_{\varkappa \tau}-n^{2}\right)
\end{aligned}
$$

and the trace as the first integral

$$
\frac{1}{3} \theta^{2}=\sigma^{2}+3 a^{2}+\frac{1}{2}\left(n^{\delta \mu} n_{\delta \mu}-\frac{1}{2} n^{2}\right)+\mu+\Lambda
$$

which is a generalisation of the Friedmann equation.

From (3.4) there follows

$$
\partial_{0}\left(\sigma^{2}\right)=-2 \theta \sigma^{2}+2 \sigma^{\beta \delta} \varepsilon^{\varkappa \tau}{ }_{(\beta} n_{\delta) \varkappa} a_{\tau}-2 \sigma_{\beta}^{\delta} n_{\delta}^{\mu} n_{\mu}^{\beta}+n n_{\beta}^{\mu} \sigma_{\mu}^{\beta} .
$$

Using this, (1.3) and (2.11), (2.12), we can verify that (3.5) is a first integral.

From the Gauss-Codacci equations we can find the Ricci tensor $R_{\alpha \beta}^{*}$ of the three-surfaces of homogeneity

$$
\begin{array}{r}
-R_{\alpha \beta}^{*}=\partial_{0} \sigma_{\alpha \beta}+\theta \sigma_{\alpha \beta}-2 \sigma_{\varkappa(\beta} \varepsilon_{\alpha) \tau}^{\varkappa} \Omega^{\tau}+\frac{2}{3} \delta_{\alpha \beta}\left(\frac{1}{3} \theta^{2}-\sigma^{2}-\mu-\Lambda\right) \\
=2 \varepsilon^{\tau \varkappa}{ }_{(\alpha} n_{\beta) \tau} a_{\varkappa}-2 n_{\alpha \delta} n_{\beta}^{\delta}+n n_{\alpha \beta}+\delta_{\alpha \beta}\left(2 a^{2}+n_{\varkappa \tau} n^{\varkappa \tau}-\frac{1}{2} n^{2}\right)
\end{array}
$$

the equivalence of the two expressions following from (3.4). Hence the scalar curvature of the three-surfaces of homogeneity is

$$
\begin{aligned}
-R^{*} & =-R_{\alpha}^{* \alpha}=2\left(\frac{1}{3} \theta^{2}-\sigma^{2}-\mu-\Lambda\right) \\
& =n_{\varkappa \tau} n^{\varkappa \tau}-\frac{1}{2} n^{2}+6 a^{2}
\end{aligned}
$$


the first being a special case of an equation due to RAYCHAUDHURI [17] which implies another relation of RAYCHAUDHURI's:

$$
\partial_{0}\left(R^{*}-2 \sigma^{2}\right)=-\frac{2}{3} \theta\left(R^{*}-6 \sigma^{2}\right) .
$$

We can now define the Weyl tensor $C_{a b c d}$ (which is the part of the curvature not determined by the local matter, and so represents the free gravitational field [20]), by

$$
\begin{aligned}
& R_{a b c d}=C_{a b c d}-g_{a[d} R_{c] b}-g_{b[c} R_{d] a}-\frac{R}{3} g_{a[c} g_{d] b}, \\
& C_{a b c d}=C_{[a b][c d]} ; \quad C_{b c a}^{a}=0=C_{a[b c d]} .
\end{aligned}
$$

We may further split this into its "electric" and "magnetic" components

$$
E_{a b}:=-C_{a p b q} u^{p} u^{q} ; \quad H_{a b}:=-\frac{1}{2} C_{a}^{p q r} \eta_{q r b s} u_{p} u^{s}
$$

each of which has five independent components since $(3.10 \mathrm{~b})$ implies $H_{a b}=H_{(a b)} ; E_{a b}=E_{(a b)} ; E_{a}^{a}=0=H_{a}^{a} ; H_{a b} u^{b}=0=H_{a b} u^{b}$.

Substituting $u^{a}$ in (3.1) we derive the expressions

$$
\begin{aligned}
& E_{\alpha \beta}=\partial_{0}\left(\sigma_{\alpha \beta}\right)-2 \sigma_{\varkappa(\alpha} \varepsilon_{\beta) \tau} \Omega^{\tau}+\sigma_{\alpha \gamma} \sigma_{\beta}^{\gamma}+\frac{2}{3} \theta \sigma_{\alpha \beta}-\frac{2}{3} \delta_{\alpha \beta} \sigma^{2}, \\
& H_{\alpha \beta}=\left(\sigma_{\varkappa \tau} n^{\varkappa \tau}\right) \delta_{\alpha \beta}+\frac{1}{2} n \sigma_{\alpha \beta}-3 \sigma_{\mu(\alpha} n_{\beta}^{\mu}-\varepsilon^{\delta \gamma}{ }_{(\beta} \sigma_{\alpha) \gamma} a_{\delta} .
\end{aligned}
$$

The Eqs. $(3.2,3,4,5,7 \mathrm{~b}, 8 \mathrm{~b}$, and 13) are written out in full in Appendix I with the tetrad choice (2.14), and those that are useful for the case $n_{\alpha}^{\alpha}=0$, in Appendix II with the specialisation (2.19). One consequence [14] is that the curvature $R^{*}$ cannot be zero if the group is Class B or (in Class A) of type II, and can be positive only if the group is of type IX.

We can define an "average distance" $l(t)$ [3], corresponding to the function $R(t)$ in the Friedmann world models, by the equation

$$
l \cdot l=\theta / 3 \text {. }
$$

$l$ is thus determined up to a factor by the volume behaviour of the space. Then the quantities

$$
H_{0}:=\left.\frac{1}{3} \theta\right|_{0}=\left.\frac{l}{l}\right|_{0} \text { and } q_{0}:=\left.\frac{-\left(\theta^{*}+\frac{1}{3} \theta^{2}\right)}{3 H_{0}^{2}}\right|_{0}=-\left.\frac{3 \ddot{l} l}{i^{2}}\right|_{0}
$$

where $t=t_{0}$ is a given time in the world model, correspond to the Hubble constant and the deceleration parameter at that time and are obtained from measurements of the volume expansion alone (thus differing slightly from the similar quantities defined by SAUnders [14]). Choosing a tetrad in the principal directions of the shear tensor we may write $\left.\theta_{\mu \nu}\right|_{0}=\operatorname{diag}\left(H_{0}\left(1+\varepsilon_{1}\right), H_{0}\left(1+\varepsilon_{2}\right), H_{0}\left(1+\varepsilon_{3}\right)\right)$ where $\varepsilon_{1}+\varepsilon_{2}+\varepsilon_{3}=0$; 
thus $H_{0}\left(1+\varepsilon_{v}\right)$ is the Hubble constant in the principal shear direction $\boldsymbol{e}_{v}$, and can be obtained from the redshift in that direction. It then follows that the relative root-mean-square deviation from isotropy, defined by $\varepsilon^{2}:=\frac{1}{3} \Sigma\left(\varepsilon_{\nu}^{2}\right)$ is related to the shear by $\frac{2}{3} \sigma^{2}=\varepsilon^{2}\left(H_{0}\right)^{2}$. Raychaudhuri's Eqs. (3.2), (3.8a) now give the relations

and

$$
\Lambda=\left.\frac{1}{2} \mu\right|_{0}+\left.\frac{3}{2} p\right|_{0}-3 H_{0}^{2} q_{0}+3 \varepsilon^{2} H_{0}^{2}
$$

$$
\left.R^{*}\right|_{0}=3\left(\left.\mu\right|_{0}+\left.p\right|_{0}-2 H_{0}^{2}\left(1+q_{0}\right)+3 \varepsilon^{2} H_{0}^{2}\right)
$$

which generalise the usual relations in isotropic world models [21]. $H_{0}, q_{0}$ and $\varepsilon^{2}$ having been observationally determined, these are relations between $\Lambda,\left.R^{*}\right|_{0},\left.\mu\right|_{0}$ and $\left.p\right|_{0}$. If $\Lambda=\left.p\right|_{0}=0$, as seems plausible, we find

and

$$
\left.\mu\right|_{0}=6 H_{0}^{2}\left(q_{0}-\varepsilon^{2}\right)
$$

$$
\left.R^{*}\right|_{0}=6 H_{0}^{2}\left(2 q_{0}-1\right)-9 \varepsilon^{2} H_{0}^{2} \text {. }
$$

\section{Class A Solutions}

We consider solutions of the field equations in which

$$
a^{\beta}=0
$$

so that the Jacobi Eqs. (2.13) are satisfied. These are of Class A (see Section 2). If we make the tetrad choice (2.14), it follows from the $(0 v)$ equations, (3.3), that the tetrad vectors are shear eigenvectors, or, if for instance $n_{2}=n_{3}$, may be chosen as such without violating (2.14). Then the $(\alpha \beta)$ equations, $(\alpha \neq \beta)$, together with the Jacobi identities (2.12), show that these shear eigenvectors are Fermi-propagated, or, if for instance $\theta_{2}=\theta_{3}$, may be chosen as such without violating (2.14). Thus we have

$$
\Omega^{a}=0 ; \quad \theta_{\mu \nu}=\operatorname{diag}\left(\theta_{1}, \theta_{2}, \theta_{3}\right) .
$$

From the Eqs. (3.7) it follows that the triad thus chosen is a triad of Ricci eigenvectors of the three-surfaces orthogonal to $u^{a}$.

Lemma 4.1. In a spacetime of Class $A$ there exists an orthonormal tetrad $\left\{e_{a}\right\}$ with $u=e_{0}$ and such that the vectors $\left\{e_{\beta}\right\}$ are Fermi-propagated shear eigenvectors of the flow congruence and Ricci eigenvectors of the surfaces of homogeneity. The Weyl tensor is type I (or its specialisations $D$ or $O)$.

With this tetrad the remaining Jacobi identities are the three timederivative equations for the $n_{\gamma}$, and the remaining field equations are the (l 1 1), ( $\left.\begin{array}{ll}2 & 2\end{array}\right)$ and $\left(\begin{array}{ll}3 & 3\end{array}\right)$ equations together with the first integral (3.5).

Having chosen the tetrad as above we can choose comoving coordinates $x^{a}$, with $x^{0}=t$, such that the coordinate-tetrad relations

$$
\boldsymbol{e}_{a}=e_{a}^{i} \frac{\partial}{\partial x^{i}}
$$


have the form

$$
\begin{aligned}
& \boldsymbol{e}_{0}=\frac{\partial}{\partial x^{0}}, \\
& \boldsymbol{e}_{3}=C\left(x^{0}\right) \frac{\partial}{\partial x^{3}}, \\
& \boldsymbol{e}_{1}=A\left(x^{0}\right)\left[\left(1-N_{1} N_{2} g^{2}\left(x^{3}\right)\right)^{1 / 2} \frac{\partial}{\partial x^{1}}-N_{2} g\left(x^{3}\right) \boldsymbol{Z}\right] \\
& \boldsymbol{e}_{2}=B\left(x^{0}\right)\left[N_{1} g\left(x^{3}\right) \frac{\partial}{\partial x^{1}}+\left(1-N_{1} N_{2} g^{2}\left(x^{3}\right)\right)^{1 / 2} \boldsymbol{Z}\right] \\
& \boldsymbol{Z}:=\left(1-N_{2} N_{3} S^{2}\left(x^{1}\right)\right)^{-1 / 2}\left(\frac{\partial}{\partial x^{2}}+N_{3} S\left(x^{1}\right) \frac{\partial}{\partial x^{3}}\right),
\end{aligned}
$$

where $N_{1}, N_{2}, N_{3}$ are constants and

$$
\begin{aligned}
& n_{1}=\frac{N_{1} B C}{A}, n_{2}=\frac{N_{2} C A}{B}, n_{3}=\frac{N_{3} A B}{C} ; \\
& \frac{\partial^{2} S}{\left(\partial x^{1}\right)^{2}}=-N_{2} N_{3} S\left(x^{1}\right) ; \quad \frac{\partial^{2} g}{\left(\partial x^{3}\right)^{2}}=-N_{1} N_{2} g\left(x^{3}\right) ; \\
& \lim _{x^{1} \rightarrow 0}\left(\frac{\partial S}{\partial x^{1}}\right)=1 ; \\
& \lim _{x^{3} \rightarrow 0}\left(\frac{\partial g}{\partial x^{3}}\right)=-1 \text {. }
\end{aligned}
$$

The coordinates are regular if $\lim _{x^{1} \rightarrow 0}(S)=0$ and $\lim _{x^{3} \rightarrow 0}(g)=0$ and singular if for example $\lim _{x^{1} \rightarrow 0}(S)=1$ or $\lim _{x^{3} \rightarrow 0}(g)=1$. We can easily check that all the $\gamma_{b c}^{a}$ are zero, except the $n_{\alpha}$ given above and

$$
\theta_{1}=-\frac{\partial}{\partial x^{0}}(\log A), \quad \theta_{2}=-\frac{\partial}{\partial x^{0}}(\log B), \quad \theta_{3}=-\frac{\partial}{\partial x^{0}}(\log C) \text {. }
$$

Thus we have satisfied (2.14), (4.1) and (4.2), and $n_{\gamma}$ may be chosen arbitrarily in an initial surface $\{t=$ constant $\}$. The Jacobi Eqs. (2.12) are, by (4.6) and (4.7), satisfied with arbitrary choice of $\theta_{\gamma}(t)$. Thus any spacetime of Class A may be described by coordinates related to the tetrad of Lemma 4.1 by (4.4) and (4.6).

Theorem 4.2. In a spacetime of Class $A$, there exist coordinates $x^{a}$ such that the metric tensor takes the form

$$
\begin{aligned}
g_{00}= & -1, \quad g_{0 \gamma}=0 \\
g_{11}= & X^{2}\left(x^{0}\right)\left(1-N_{1} N_{2} g^{2}\left(x^{3}\right)\right)+Y^{2}\left(x^{0}\right) \\
g_{12}= & g\left(x^{3}\right)\left(1-N_{1} N_{2} g^{2}\left(x^{3}\right)\right)^{1 / 2}\left(1-N_{2} N_{3} S^{2}\left(x^{1}\right)\right)^{1 / 2} \\
& \cdot\left(N_{2} Y^{2}\left(x^{0}\right)-N_{1} X^{2}\left(x^{0}\right)\right), \\
g_{22}= & X^{2}\left(x^{0}\right)\left(N_{1} g\left(x^{3}\right)\right)^{2}\left(1-N_{2} N_{3} S^{2}\left(x^{1}\right)\right) \\
& +Y^{2}\left(x^{0}\right)\left(1-N_{2} N_{3} S^{2}\left(x^{1}\right)\right)\left(1-N_{1} N_{2} g^{2}\left(x^{3}\right)\right) \\
& +Z^{2}\left(x^{0}\right)\left(N_{3} S\left(x^{1}\right)\right)^{2}, \\
g_{13}= & 0, \quad g_{23}=-Z^{2}\left(x^{0}\right) N_{3} S\left(x^{1}\right), \quad g_{33}=Z^{2}\left(x^{0}\right),
\end{aligned}
$$

where $S\left(x^{1}\right), g\left(x^{3}\right)$ are determined from the constants $N_{1}, N_{2}, N_{3}$ by (4.6). 
These are in fact the coordinates used in the relations (4.4), where to find the metric we have inverted these relations and used (2.2), writing $X=1 / A, Y=1 / B, Z=1 / C$. The remaining field equations and conservation equations yield

$$
\left.\begin{array}{rl}
\frac{\mu}{\mu+p} & =-(\log X Y Z)^{\cdot} \\
\Lambda+\frac{1}{2}(\mu & -p) \\
& =\frac{\ddot{X}}{X}+\frac{\dot{X} \dot{Y}}{X Y}+\frac{\dot{X} \dot{Z}}{X Z}+\frac{1}{2}\left(\frac{N_{1} X}{Y Z}\right)^{2}-\frac{1}{2}\left(\frac{N_{2} Y}{X Z}-\frac{N_{3} Z}{X Y}\right)^{2} \\
& =\frac{\ddot{Y}}{Y}+\frac{\dot{Y} \dot{X}}{Y X}+\frac{\dot{Y} \dot{Z}}{Y Z}+\frac{1}{2}\left(\frac{N_{2} Y}{X Z}\right)^{2}-\frac{1}{2}\left(\frac{N_{3} Z}{X Y}-\frac{N_{1} X}{Y Z}\right)^{2} \\
& =\frac{\ddot{Z}}{Z}+\frac{\dot{Z} \dot{X}}{Z X}+\frac{\dot{Z} \dot{Y}}{Z Y}+\frac{1}{2}\left(\frac{N_{3} Z}{X Y}\right)^{2}-\frac{1}{2}\left(\frac{N_{1} X}{Y Z}-\frac{N_{2} Y}{X Z}\right)^{2}
\end{array}\right\}
$$

and the first integral is

$$
\begin{gathered}
\mu+\Lambda=\frac{\dot{X} \dot{Y}}{X Y}+\frac{\dot{Y} \dot{Z}}{Y Z}+\frac{\dot{Z} \dot{X}}{Z X}+\frac{1}{2}\left(\frac{N_{2} N_{3}}{X^{2}}+\frac{N_{1} N_{3}}{Y^{2}}+\frac{N_{2} N_{1}}{Z^{2}}\right) \\
-\frac{1}{4}\left(\left(\frac{N_{3} Z}{X Y}\right)^{2}+\left(\frac{N_{1} X}{Y Z}\right)^{2}+\left(\frac{N_{2} Y}{Z X}\right)^{2}\right) .
\end{gathered}
$$

The Eqs. (4.9) have known analytic solutions only for the vacuum case $[5,22]$ and for groups of type $\mathrm{I}\left(n_{a}=0\right)$. This latter case has been investigated in the case of dust by Heckmanx and Schücking [6], RaYCHAUdHuRI [23], Robinson [24], and Saunders [14] (who included the $\Lambda$-term); for perfect fluids by Thorne [25], Doroshrevich [26], SteWART [2], and JACOBS [27]; and for vacuum spaces by TAUB [5], KASNER [28], Dautcourt et al. [29], and others. The type IX case has been investigated by BeHr [12] and SHePley [13].

The simplest cases in Class A which have not been analytically integrated are those with local rotational symmetry [1, 2] (see Section 7). Unless these can be integrated analytically, it is unlikely that any more complex or general cases can be so integrated. The difficulty arises from the characteristic cross-terms in $X, Y$ and $Z$ which occur when any of the $N_{\beta}$ (i.e. $n_{\beta}$ ) are non-zero. A further possibly-integrable case arises when these cross-terms can be made to vanish by use of the specialisation (2.19), i.e. when $n=0$. This and certain other special cases are discussed in subsequent sections.

\section{Class B Solutions}

We now consider solutions in which the group is of Class B, i.e. using the tetrad choice (2.14)

$$
a \neq 0
$$


The Jacobi identities (2.13) are satisfied if and only if

$$
n_{1}=0 \text {. }
$$

The Jacobi identities (2.11) now imply

$$
\Omega^{3}=\sigma_{12}, \quad \Omega^{2}=-\sigma_{13} .
$$

As well as the first integral (3.5) we have three non-trivial constraint Eqs. (3.3), and these are all conserved under the time-propagation.

The consistency conditions are somewhat more involved than in Class A. First, we note from (3.3) that either

or

$$
a^{\beta} \text { is a shear eigenvector } \Leftrightarrow \sigma_{12}=\sigma_{13}=0
$$

$a^{\beta}$ is not a shear eigenvector $\Leftrightarrow \sigma_{12} \sigma_{13} \neq 0 \Rightarrow n_{2} n_{3}+9 a^{2}=0 .(5.4 \mathrm{~b})$

Using definition (2.18) we see that

Theorem 5.1. The only Class $B$ spacetime in which $a^{\beta}$ is not necessarily a shear eigenvector is that with a group of type $\mathrm{VI}_{h}$ with $h=-1 / 9$.

If $n_{2}=n_{3}$ on an open set, then $\partial_{0}\left(n_{2}-n_{3}\right)=0$ shows, using (2.12), that either $n_{2}=n_{3}=0$, or $n_{2}+n_{3} \neq 0$ and $\theta_{2}=\theta_{3}$. (2.12) further yields $\sigma_{23}=0$, and $\partial_{0}\left(\sigma_{23}\right)=0 \Rightarrow \sigma_{12} \sigma_{13}=0$. Using this with (5.4) and the $\left(\begin{array}{ll}0 & 1\end{array}\right)$ equation, which is

$$
\theta_{1}=\frac{1}{2}\left(\theta_{2}+\theta_{3}\right)
$$

we find we have a Robertson-Walker model, which we prove in Section 7 to admit a group of type $\mathrm{V}$.

Lemma 5.2. $A$ spacetime of Class $B$ has $n_{2}=n_{3}$ on an open neighbourhood if and only if there is a group of type $V$.

We call these spacetimes of Case Ba. We may choose the tetrad to satisfy (2.16) and so that the vectors $\left\{\boldsymbol{e}_{\beta}\right\}$ are Ricci eigenvectors of the three-surfaces orthogonal to $u_{\beta}$ and Fermi-propagated eigenvectors. This is because when $n_{2}=n_{3}=0$ we have the freedom of a rotation $\phi\left(x^{0}\right)$ in the $\boldsymbol{e}_{2} / \boldsymbol{e}_{3}$ plane which we use to make $\Omega^{1}=0$ everywhere and $\sigma_{23}=0$ initially; then the time derivative equations show that $\sigma_{\mathbf{2}}=0$ everywhere. Then (5.5) holds. So we have

Case $\mathrm{Ba}: n_{2}=n_{3}$ on an open set $\Leftrightarrow$ there is a group of type $\mathrm{V} \Leftrightarrow$ we can satisfy (2.14), (5.1) with $n_{\beta}=\Omega_{\beta}=0=\sigma_{\alpha \beta}(\alpha \neq \beta)$ and (5.5) holds.

If $n_{2} \neq n_{3}$ on any open set, we can solve (2.12) for $\Omega^{1}$ in terms of $\sigma_{23}$, and we also have $\left(\begin{array}{ll}0 & 1\end{array}\right)$, as:

$$
\begin{aligned}
& \Omega_{1}=\left(n_{2}+n_{3}\right) \sigma_{23} /\left(n_{3}-n_{2}\right), \\
& \theta_{1}=\frac{1}{2}\left(\theta_{2}+\theta_{3}\right)-\left(n_{2}-n_{3}\right) \sigma_{23} / 2 a .
\end{aligned}
$$

If $\sigma_{23}=0$ on an open neighbourhood, (5.5) holds, and if $n_{2} \neq n_{3}$, $(2.12) \Rightarrow \Omega^{1}=0$ and $\partial_{0}\left(\sigma_{23}\right)=0$ gives a first integral

$$
2 \sigma_{12} \sigma_{13}+a\left(n_{3}-n_{2}\right)=0
$$


which shows that $\sigma_{12} \sigma_{13} \neq 0$ and hence $h=-1 / 9$. [The time derivative of this equation shows, using (3.3), that $\theta_{2}\left(3\left(\sigma_{13}\right)^{2}+\left(\sigma_{12}\right)^{2}\right)+\theta_{3}\left(\left(\sigma_{13}\right)^{2}\right.$ $\left.+3\left(\sigma_{12}\right)^{2}\right)=0$ which is a new constraint showing that matter does not recede in one of the principal directions. So if $\sigma_{23}=0$ and matter expands in all directions, the group is type $V$. .]

We thus have two cases when $n_{2} \neq n_{3}$ and (5.1) holds.

Case Bbi: $\sigma_{12}=\sigma_{13}=0 ; \sigma_{23} \neq 0$. (This can be written in a new tetrad such that $n_{23} \neq 0$ and $\sigma_{23}=0$, but this makes little difference.)

Case Bbii: $\sigma_{12} \sigma_{13} \neq 0 ; h=-1 / 9$ and $n_{2}=\frac{3 a \sigma_{13}}{\sigma_{12}}, n_{3}=\frac{-3 a \sigma_{12}}{\sigma_{13}}$.

Lemma 5.3. The only Class $B$ spacetimes in which the eigenvectors of $n_{\alpha \beta}$ are shear eigenvectors are those of case $B a$.

As in Class A, we do not expect in general to find analytic solutions with $n_{2}$ or $n_{3}$ non-zero. Hence we are likely to obtain such solutions only in Case $\mathrm{Ba}$. In this case we find a first integral applicable to any perfect fluid and previously given for dust by HeckMans and Schücking [6] and Robinson [24]. This equation may be integrated in certain cases (see Section 7); in particular when $\Lambda=\mu=0$ [31].

One may also obtain solutions when the space is an L.R.S. space with a group of Bianchi type III. When $n_{\alpha}^{\alpha}=0$, the terms in the $n_{\beta}$ may again be rendered in a simpler form by use of the tetrad choice (2.19). These special cases are discussed in subsequent sections.

\section{The Case $n_{\boldsymbol{\beta}}^{\boldsymbol{\beta}}=\mathbf{0}$}

In the last two sections the field equations have been reduced to a form in which they are in general consistent. This may no longer be true when the value of some quantity [e.g. $\left(\theta_{2}-\theta_{3}\right)$ ] is constrained to be zero at all times. The constraint gives rise to further constraint equations through the time propagation [e.g. $\left.\partial_{0}\left(\theta_{2}-\theta_{3}\right)=0\right]$ : these further constraints must themselves be conserved in time giving rise to yet more constraints. Either these sets of constraints will lead to inconsistencies, or there will come a stage where the new constraints found are merely identities by virtue of the previous equations and we will have a consistent solution. In this and the following sections we will examine some such special cases.

In this section we consider $n=0$. We may choose a tetrad satisfying (2.19) with $q \geqq 0, r \geqq 0$.

In Class $A$ solutions, $a=0$ and the $(0 v)$ equations show

$$
\begin{aligned}
& q\left(\sigma_{13}-\sigma_{12}\right)+r\left(\theta_{3}-\theta_{2}\right)=0, \\
& r\left(\sigma_{13}-\sigma_{12}\right)+q\left(\theta_{3}-\theta_{2}\right)=0 .
\end{aligned}
$$

If $q=r \neq 0$, the Jacobi identities and (6.1) show that $\sigma_{\mu \nu}=0$, which is incompatible with the time development equations for the shear. If 
$0 \neq q \neq r$, then (6.1) shows $\sigma_{12}=\sigma_{13}, \theta_{2}=\theta_{3}$. Then the Jacobi identities imply

$$
\begin{aligned}
& r\left(\Omega_{2}+\Omega_{3}\right)-2 q \Omega_{1}=0, \\
& q\left(\Omega_{2}+\Omega_{3}\right)-2 r \Omega_{1}=0
\end{aligned}
$$

which shows $\Omega_{1}=\Omega_{2}+\Omega_{3}=0$ and with these restrictions the Jacobi identities further yield $\Omega_{2}=-\sigma_{12}$ and $2 r \sigma_{12}+q \sigma_{23}=0$. Taking the time derivative of this last equation and using $\left(\begin{array}{ll}0 & 2\end{array}\right)$ we find the further constraint $q\left(3 r^{2}+\left(\sigma_{12}\right)^{2}+2\left(\sigma_{23}\right)^{2}\right)=0$ from the time conservation. This proves that $r=\sigma_{12}=\sigma_{23}=0$, since $q=0, r \neq 0$ leads easily to an inconsistency.

Theorem 6.1. $n_{\beta}^{\beta}$ is not zero in any open set in a spacetime of Class $A$ invariant under a group of type VIII.

The above implies that in Class $A$ we can always set

$$
r=0=\Omega_{\beta}=\sigma_{\alpha \beta}(\alpha \neq \beta) .
$$

In the case $q \neq 0$ we have the further condition

$$
\theta_{2}=\theta_{3} \text {. }
$$

In Class $B$ solutions $r=0$ and the Jacobi identities imply $\sigma_{12}=\Omega_{3}$, $\sigma_{13}=-\Omega_{2}$. If $q \neq 0, \Omega_{1}=\sigma_{23}=0$. Since in the case $q=0$ (Case Ba) this last equation may be made true by choice of tetrad, we may assume it true in all cases. In Case Ba and Case Bbi solutions, $a^{\beta}$ is a shear eigenvector and so $\sigma_{12}=\sigma_{13}=0$, whence $(6.2 \mathrm{a})$ is still true. In Case Bbii, $a^{\beta}$ is not a shear eigenvector and the equations $\left(\begin{array}{ll}0 & 2\end{array}\right),\left(\begin{array}{ll}0 & 3\end{array}\right)$ are

$$
0=\sigma_{12}(q+3 a)=\sigma_{13}(q-3 a) .
$$

We may therefore choose the axes so that $\sigma_{12} \neq 0$, and we then have

$$
\Omega_{3}=\sigma_{12} \neq 0 \Rightarrow q=-3 a, \Omega_{2}=\Omega_{1}=\sigma_{13}=\sigma_{23}=0 .
$$

In all Class $B$, the $\left(\begin{array}{ll}0 & 1\end{array}\right)$ equation reads

$$
0=a\left(2 \theta_{1}-\theta_{2}-\theta_{3}\right)+q\left(\theta_{3}-\theta_{2}\right)
$$

which reduces in Case Bbii to

$$
\theta_{3}=\frac{1}{2}\left(\theta_{2}+\theta_{1}\right)
$$

Combining the results for the two Classes, $A$ and $B$, we find there are two families of solutions in which $n_{\beta}^{\beta}=0$, a general case characterised by (6.2a) and a special case characterised by (6.4). In either case we may choose coordinates related to the canonical tetrad (2.19) by;

$$
\begin{aligned}
& \boldsymbol{e}_{0}=\frac{\partial}{\partial x^{0}}, \\
& \boldsymbol{e}_{1}=A\left(x^{0}\right)\left(\frac{\partial}{\partial x^{1}}+f\left(x^{0}\right) \exp \left(\left(a_{0}+q_{0}\right) x^{1}\right) \frac{\partial}{\partial x^{2}}\right), \\
& \boldsymbol{e}_{2}=B\left(x^{0}\right) \exp \left(\left(a_{0}+q_{0}\right) x^{1}\right) \frac{\partial}{\partial x^{2}} \\
& \boldsymbol{e}_{3}=C\left(x^{0}\right) \exp \left(\left(a_{0}-q_{0}\right) x^{1}\right) \frac{\partial}{\partial x^{3}}
\end{aligned}
$$

\footnotetext{
Commun. math. Phys., Vol. 12
} 
where $\theta_{1}=-A^{\cdot} \mid A, \theta_{2}=-B \cdot B, \theta_{3}=-C^{\cdot} / C$ and $(A f)^{\cdot}=-2 \sigma_{12} B ; q_{0}, a_{0}$ are constants, and $q=q_{0} A, a=a_{0} A$. The corresponding metric is

$$
\begin{aligned}
d s^{2}= & -d t^{2}+\left\{X^{2}\left(x^{0}\right)+Y^{2}\left(x^{0}\right) f^{2}\left(x^{0}\right)\right\}\left(d x^{1}\right)^{2} \\
& -\left\{2 Y^{2}\left(x^{0}\right) f\left(x^{0}\right) \exp \left(-\left(a_{0}+q_{0}\right) x^{1}\right)\right\} d x^{1} d x^{2} \\
& +\left\{Y^{2}\left(x^{0}\right) \exp \left(-2\left(a_{0}+q_{0}\right) x^{1}\right)\right\}\left(d x^{2}\right)^{2} \\
& +\left\{Z^{2}\left(x^{0}\right) \exp \left(2\left(q_{0}-a_{0}\right) x^{1}\right)\right\}\left(d x^{3}\right)^{2},
\end{aligned}
$$

where we have defined $X=1 / A, Y=1 / B, Z=1 / C$. In Case Bbii we can integrate the $\partial_{0} \sigma_{12}$ equation to give $\sigma_{12}=b B^{2} C$ and $f\left(x^{0}\right)$ $=-2 b \int \frac{X}{Y^{3} Z} d x^{0}$ where $b$ is a constant. $b \neq 0 \Leftrightarrow q=-3 a$ and $\sigma_{12} \neq 0 \neq f\left(x^{0}\right)$.

The remaining field equations and their first integral now read

$$
\begin{aligned}
\frac{\mu}{\mu+p} & =-(\log X Y Z)^{\circ} \\
\Lambda+\frac{1}{2}(\mu-p) & =\frac{\ddot{X}}{X}+\frac{\dot{X} \dot{Y}}{X Y}+\frac{\dot{X} \dot{Z}}{X Z}-\frac{2\left(a_{0}^{2}+q_{0}^{2}\right)}{X^{2}}+\frac{2 b^{2}}{Y^{4} Z^{2}} \\
& =\frac{\ddot{Y}}{Y}+\frac{\dot{Y} \dot{X}}{Y X}+\frac{\dot{Y} \dot{Z}}{Y Z}-\frac{2\left(a_{0}^{2}+a_{0} q_{0}\right)}{X^{2}}-\frac{2 b^{2}}{Y^{4} Z^{2}} \\
& =\frac{\ddot{Z}}{Z}+\frac{\dot{Z} \dot{X}}{Z X}+\frac{\dot{Z} \dot{Y}}{Z Y}-\frac{2\left(a_{0}^{2}-a_{0} q_{0}\right)}{X^{2}}, \\
\mu+\Lambda+\frac{\left(3 a_{0}^{2}+q_{0}^{2}\right)}{X^{2}} & =\frac{\dot{X} \dot{Y}}{X Y}+\frac{\dot{X} \dot{Z}}{X Z}+\frac{\dot{Z} \dot{Y}}{Z Y}+\frac{2 b^{2}}{Y^{4} Z^{2}} .
\end{aligned}
$$

Theorem 6.2. For all spacetimes satisfying (1.1), (1.2) and (1.4) in which $n_{\beta}^{\beta}=0$, there exists an orthonormal tetrad satisfying (2.19) and coordinates $x^{a}$ related to this tetrad by (6.5). The metric is then given by (6.6) and the field equations by (6.7), with the constraint $(6.3 \mathrm{~b})$.

This form is generally simpler than the other tetrad choice (2.14). There are the following subcases.

Class $A: a=0, b=0$.

$q=0 \Leftrightarrow$ the group is type I. These spaces have isotropic threespaces (see Section 7) and may be integrated for many types of matter (see Ref. [27]).

$q \neq 0 \Rightarrow$ the group is type $\mathrm{VI}_{0}$ and $\theta_{2}=\theta_{3}$. There are two independent $\theta_{v}$ but the shear equation is more complex than in type $\mathrm{V}$, where (5.5) holds.

Class $B: a \neq 0$.

$q=0 \Leftrightarrow$ type $\mathrm{V} \Leftrightarrow(5.5)$ holds. The three-spaces are isotropic and we may reduce the shear equations to a first integral (see Section 7).

$q \neq 0, b=0 \Rightarrow$ Case Bbi, group type $\mathrm{VI}_{h}$. $(6.3 \mathrm{~b})$ determines $\theta_{1}$. This is in general similar to the case of Class $\mathrm{A}$, type $\mathrm{VI}_{0}$, above. There is a special case when $h=-1 \Leftrightarrow q=a$ which is of Bianchi type III, is L.R.S. and has been integrated for several behaviours of matter [32, 33]. 
$q \neq 0, b \neq 0 \Rightarrow$ the special case $q=-3 a \Leftrightarrow 2 \theta_{3}=\theta_{1}+\theta_{2}$ which is of case Bbii; there is now a cross-term of the more complex type found in (4.9) due to the off-diagonal component of shear.

The simplest cases with the more difficult cross-terms are those of type II which are L.R.S. and the type $\mathrm{VI}_{h}\left(n_{\beta}^{\beta}=0\right)$ case with $\sigma_{12} \neq 0$ just discussed. All other solutions have in general several such crossterms and are unlikely to be integrated before the ones listed above.

Most of these cases have been integrated in the vacuum case $\left(p=\mu=\Lambda=0\right.$ ) (see Section 8) and Bianchi types I and III $\left(n_{\beta}^{\beta}=0\right)$ have been integrated for several types of fluid. None of the rest have been integrated even for dust $(p=\Lambda=0)$. A special situation arises when $p=\mu$ ("stiff" matter), when apart from types I and III we may integrate in type $\mathrm{V}$ (see Section 7) and type $\mathrm{VI}_{0}\left(n_{\beta}^{\beta}=0=\Lambda\right.$ ). This last solution is

$$
\begin{aligned}
d s^{2}= & X^{2}\left(-d u^{2}+\left(d x^{1}\right)^{2}\right) \\
& +2 u\left(\exp \left(-2 q_{0} x^{1}\right)\left(d x^{2}\right)^{2}+\exp \left(2 q_{0} x^{1}\right)\left(d x^{3}\right)^{2}\right)
\end{aligned}
$$

where $X^{2}=u^{(M-1) / 2} \exp \left(q_{0} u^{2}\right)$ and $\mu=M / 4 u^{2} X^{2}$. Setting $M=0$ gives the vacuum solution with $\Lambda=0$. In the case of dust the type $\operatorname{VI}_{0}\left(n_{\beta}^{\beta}=0\right)$ field equations reduce to

$$
\begin{aligned}
2 X Y \dot{Y} & =t \\
t^{2}\left(2 Y \ddot{Y}+\dot{Y}^{2}\right)+Y^{4} \dot{Y}^{2} & =0
\end{aligned}
$$

to which we could find only the special solution $Y^{2}=X=t$. This seems to be the simplest case without a known general analytic solution.

\section{Spacetimes With Some Isotropy}

In this section we consider first those spaces satisfying (1.1), (1.2) and (1.4) which are also locally rotationally symmetric (see Refs. [1] and [2] for detailed discussion). These are spaces in which there is a group of motions $G_{r}$ with $r=4$ or 6 , multiply-transitive on the three-surfaces orthogonal to the fluid flow vector $u^{a}$, and having at least one threeparameter subgroup simply transitive on these surfaces. If $r=6$, the space is a Robertson-Walker (isotropic) space; if $r=4$ the space is partially isotropic and has non-vanishing shear.

Each point $p$ is invariant under a subgroup of $G_{r}$, the isotropy group of $p$, which is one-dimensional if $r=4$ and three-dimensional if $r=6$. This group induces a group of rotations in the tangent space $T_{p}$ which leaves invariant all quantities defined covariantly at $p$ by the curvature tensor and its derivatives. It follows (cf. $[1,2])$ that if we choose a tetrad with $\boldsymbol{e}_{0}=\boldsymbol{u}$ and $\boldsymbol{e}_{1}$ as an axis of a group of rotations at each point (unique 
if $r=4$ ), then the rotation coefficients have the form

$$
\begin{aligned}
& \theta_{\gamma \delta}=\operatorname{diag}(\alpha, \beta, \beta), \quad \Omega_{\gamma}=(\Omega, 0,0), \\
& a_{\gamma}=\left(a_{1}, a_{2}, a_{3}\right), \quad n_{\gamma \pi}=\left(\begin{array}{rrc}
m & -a_{3} & a_{2} \\
-a_{3} & n & 0 \\
a_{2} & 0 & n
\end{array}\right),
\end{aligned}
$$

where $\partial_{2} \gamma=\partial_{3} \gamma=0$ for each coefficient $\gamma$,

and we can set $\Omega=0$ by choice of the time-propagation of the tetrad. The existence of a tetrad in which (7.1) is obeyed is in fact the necessary and sufficient condition for a fluid-filled spacetime to be L.R.S.

In Refs. [1] and [2] the tetrad was further restricted so that $n=a_{3}=0$, but in general the resulting rotation coefficients would not satisfy $(2.9 \mathrm{~b})$. In the present case where (7.1) and (2.9) hold, the Jacobi identities (2.13) are satisfied if and only if either

or

$$
a_{2}=a_{3}=0=m a_{1}
$$

$$
n=a_{1}=0 .
$$

As the group type is preserved in time we need consider only one surface of transitivity $S_{3}$.

The group $G_{4}$ or $G_{6}$ is multiply transitive on spacelike surfaces $S_{3}$, with a subgroup $G_{3}$ simply transitive on these surfaces; it is possible that there is another subgroup $G_{3}{ }^{\prime}$ also simply transitive on $S_{3}$. In that case there will be a position-dependent rotation relating the tetrads which generate the reciprocal groups of these groups of motions. Each of the tetrads must satisfy (2.9). If $\boldsymbol{e}_{1}$ is chosen as the axis of rotation at each point (unique if $r=4$; not unique if $r=6$ ) and $\phi$ as its magnitude we may verify directly from this requirement that $a_{\alpha}, n_{\alpha \beta}$ have the form $(7.1 \mathrm{~b})$. We also find that $m, a_{1}$ and the quantity

$$
T:=4\left(\left(a_{2}\right)^{2}+\left(a_{3}\right)^{2}\right)-n m
$$

are left invariant by the rotation. It is therefore possible to classify the solutions of (7.2) by the values of these quantities $m, a_{1}$ and $T$. The result is shown in Table 3.

The invariance of $m, a_{1}$ and $T$ permits only the following non-trivial transformations between these possible L.R.S. cases (where primed and unprimed refer to after and before the transformation respectively):

a) (7.2a) satisfied before and after the transformation, $T=m=0$, and we are only altering $n$. This carries groups of types $I$ and $V$ into groups of types $\mathrm{VII}_{0}$ and $\mathrm{VII}_{h}$ respectively. The transformation has the form

$$
\begin{aligned}
\partial_{1} \phi & =n^{\prime}-n ; \quad \partial_{2} \phi=\partial_{3} \phi=0, \\
a^{\beta}=a^{\prime \beta} & =(a, 0,0) ; \quad n_{\alpha \beta}=\operatorname{diag}(0, n, n) ; \quad n_{\alpha \beta}^{\prime}=\operatorname{diag}\left(0, n^{\prime}, n^{\prime}\right) .
\end{aligned}
$$


Table 3. All spatially homogeneous models satisfying (1.4) which are L.R.S., classified by the values of the parameters $a_{1}, m$ and $T$

\begin{tabular}{|c|c|c|c|c|c|}
\hline$a_{1}$ & $m$ & $T$ & Group type & $n$ & $a_{2}^{2}+a_{3}^{2}$ \\
\hline \multirow[t]{3}{*}{0} & 0 & 0 & $I$ & 0 & 0 \\
\hline & & & $\mathrm{VII}_{0}$ & + & 0 \\
\hline & & + & $\operatorname{III}\left(n_{\beta}^{\beta}=0\right)$ & 0 & + \\
\hline \multirow[t]{2}{*}{$\neq 0$} & 0 & 0 & $V$ & 0 & 0 \\
\hline & & & $\mathrm{VII}_{h}$ & + & 0 \\
\hline \multirow[t]{4}{*}{0} & $\neq 0$ & 0 & II & 0 & 0 \\
\hline & & + & VIII & + & 0 \\
\hline & & & $\operatorname{III}\left(n_{\beta}^{\beta} \neq 0\right)$ & 0 & + \\
\hline & & - & IX & - & 0 \\
\hline
\end{tabular}

b) $(7.2 \mathrm{~b})$ is satisfied before and after the transformation, $T>0$, and we alter only the relative magnitudes of $a_{2}, a_{3}$. The transformation has the form

$$
\begin{array}{ll}
\partial_{1} \phi=0 ; & \partial_{2} \phi=2 a\left(\sin \left(\phi+\psi^{\prime}\right)-\sin \psi\right) ; \\
\partial_{3} \phi=2 a\left(\cos \psi-\cos \left(\phi+\psi^{\prime}\right)\right)
\end{array}
$$

where $a^{\beta}=(0, a \cos \psi, a \sin \psi), a^{\prime \beta}=a\left(0, \cos \psi^{\prime}, \sin \psi^{\prime}\right)$ and $n_{\alpha \beta}$ is given by $(7.1 \mathrm{~b})$ for each case. This transforms one group of Bianchi type III into another.

c) (7.2a) satisfied before and (7.2b) after the rotation, $m \neq 0, T>0$. This transforms groups of type VIII into type III (and its inverse does the reverse). The transformation has the form

$$
\left.\begin{array}{rl}
a^{\beta} & =(0,0,0) ; \quad a^{\prime \beta}=\frac{1}{2} b c(0, \cos \psi, \sin \psi) ; \\
n_{\alpha \beta} & =\operatorname{diag}\left(-c^{2}, b^{2}, b^{2}\right) ; \\
n_{\alpha \beta}^{\prime} & =\left(\begin{array}{ccc}
-c^{2} & -\frac{1}{2} b c \sin \psi & \frac{1}{2} b c \cos \psi \\
-\frac{1}{2} b c \sin \psi & 0 & 0
\end{array}\right)
\end{array}\right\}
$$

where $b \neq 0 \neq c$ and $\partial_{1} \phi=-b^{2} ; \quad \partial_{2} \phi=b c \sin (\phi+\psi)$;

$$
\partial_{3} \phi=-b c \cos (\phi+\psi) \text {. }
$$

In all the above the quantities $\left(a, n, n^{\prime}, \psi, \psi^{\prime}, b, c\right)$ used have arbitrary values in the chosen hypersurface. 
Thus we can summarise the L.R.S. cases as follows; (7.1) holds in each case.

First suppose $r=4$. In Class $A:(7.2 \mathrm{a})$ is satisfied.

$m=0 \Leftrightarrow$ the space is invariant under a simply transitive group of type $\mathrm{I}$ and a one-parameter family of groups of type $\mathrm{VII}_{0}$, related by (7.3). As this is a special case of type I, it can be integrated.

$m \neq 0 \Leftrightarrow$ the space is invariant under a simply transitive group of type IX, II, or VIII according as $n>0, n=0, n<0$; in the last case $T>0$ and the space is also invariant under a one-parameter family of groups of type III $\left(n_{\beta}^{\beta} \neq 0\right)$. These are the cases of Class III in Refs. [1] and [2]; none has been analytically integrated except for vacuum. The last case gives the only spaces invariant under groups of Class A and Class B.

In Class $B$, if (7.2a) holds, $m=0$ and we may use (7.3) to make $n=0$. By (5.5) the space is then Robertson-Walker and so invariant under a $G_{6}$. Thus in the case $r=4, \sigma \neq 0,(7.2 \mathrm{~b})$ holds.

$m=0 \Leftrightarrow$ the space is invariant under a one-parameter family of simply transitive groups of type III $\left(n_{\beta}^{\beta}=0\right)$ related by (7.4). This case is analytically integrable [32,33] and is in class II of Refs. [1] and [2]. In fact, if the group is type III with $n_{\beta}^{\beta}=0$ the space is necessarily L.R.S.

$m \neq 0 \Leftrightarrow$ the space is invariant under a one-parameter family of simply transitive groups of Bianchi type III $\left(n_{\beta}^{\beta} \neq 0\right)$ and a simply transitive group of type VIII, related by (7.5). This is the case referred to in Class $\mathrm{A}$ above.

Now suppose $r=6$.

The space is Robertson-Walker and so invariant under a group of type $\mathrm{I}$ (or $\mathrm{VII}_{0}$ ), IX, or $\mathrm{V}$ (or $\mathrm{VII}_{h}$ ); this will be shown later in this section ${ }^{6}$. The isotropy group at each point is the rotation group $O_{3}$ (Bianchi type IX). This group permutes some of the simply transitive subgroups amongst themselves. Consideration of its action shows that the following L.R.S. cases with $\sigma=0$ (Robertson-Walker cases) can occur. We have $\theta_{\beta}=\theta / 3, \Omega=0$, and

$R^{*}=0 \Leftrightarrow a^{\beta}=m=0$. These spaces are invariant under a simply transitive group of type I; and, for each value of $n$, a three-parameter family of groups of type VII $_{0}$.

$R^{*}<0 \Leftrightarrow a^{\beta} \neq 0, m=0$. The space is invariant under a threeparameter family of groups of type $\mathrm{V}$ and, for each value of $n$ (i.e. for each value of $h$ ), a three-parameter family of groups of type $\mathrm{VII}_{h}$.

If $R^{*}>0$, the reciprocal group to the group of motions of type IX simply transitive on $S_{3}$ is also a group of motions of type IX simply

${ }_{6}$ This was also discovered by GRISHchuk [34]. 
transitive on the $S_{3}$. These two groups commute, so generating a sixparameter group of motions $O_{3} \times O_{3} \cong O_{4}$. This is the full group of motions of a three-space of constant positive curvature and we cannot find any other two commuting groups $G_{3}$ each simply transitive on the manifold ? . So we have

$R^{*}>0 \Leftrightarrow a^{\beta}=0, m=n \neq 0$. The space is invariant under two simply transitive groups of type IX; these groups are reciprocal groups.

It should be noted that the Robertson-Walker cases are not given here in the usual coordinates, but by the appropriate specialisation of the coordinates of Theorems 4.2 and 6.2 .

All the L.R.S. solutions listed above are self-consistent. The only fluid L.R.S. solution spatially homogeneous on surfaces orthogonal to $u^{a}$ which does not occur here is case I of KANTOwskI and SAcHs [32], which is the only fluid solution in which a group of motions multiply transitive on spacelike three-surfaces does not have a subgroup $G_{3}$ simply transitive in these surfaces.

In all the L.R.S. cases the quadric defined by $\theta_{\mu \nu}$ is necessarily isotropic about some axis (chosen here as $\boldsymbol{e}_{1}$ ). Conversely we may ask; what spaces result if the expansion quadric is isotropic about some axis?

In Class A suppose $\theta_{2}=\theta_{3}$. Time conservation of this implies $\left(n_{2}+n_{3}\right)\left(n_{2}+n_{3}-n_{1}\right)=0$. If $n_{2}=n_{3}$ we have L.R.S. solutions. If $n_{2} \neq n_{3}$ then $n_{1}=n_{2}+n_{3}$. The time derivative of this equation implies $n_{1}=0$ or $\sigma=0$ (which is inconsistent with $n_{2} \neq n_{3}$ ). So apart from L.R.S. cases there is only the solution of type $\mathrm{VI}_{0}$ with $n_{\beta}^{\beta}=0$ in which $\theta_{2}=\theta_{3}$ necessarily.

In case $\mathrm{Ba}$, partial isotropy implies a Robertson-Walker space.

In case $\mathrm{Bbi}$, we may choose the shear eigenvectors as axes with $a^{\beta}=(a, 0,0)$; we find that if $\theta_{2}=\theta_{3}$ we have a Robertson-Walker space. If $\theta_{1}=\theta_{2}$, then $\left(\begin{array}{ll}0 & 1\end{array}\right)$ implies $a=n_{23}$. The time derivative of this is $\Omega_{1}\left(n_{22}-n_{33}\right)=0$. The only consistent solution is $\Omega_{1}=n_{22}=0$, which is an L.R.S. solution of type III (with arbitrary $n_{\beta}^{\beta}$ ). Thus Ba and Bbi lead only to L.R.S. solutions. We have been unable to solve the more complex consistency conditions for Case Bbii, but it is unlikely that any spaces of this kind with partially isotropic shear exist.

${ }^{7}$ If a surface $S_{3}$ is regarded as given by embedding the unit three-sphere in a Euclidean four-dimensional space $R^{4}$ (with coordinates $u^{1}, u^{2}, u^{3}, u^{4}$ ) then the two simply transitive groups are generated by the two-forms

$$
\left.\begin{array}{l}
d u^{1} \wedge d u^{2}+d u^{3} \wedge d u^{4} \\
d u^{2} \wedge d u^{3}+d u^{1} \wedge d u^{4} \\
d u^{3} \wedge d u^{1}+d u^{2} \wedge d u^{4}
\end{array}\right\} \quad \text { and } \quad \begin{aligned}
& d u^{1} \wedge d u^{2}-d u^{3} \wedge d u^{4} \\
& \\
& d u^{3} \wedge d u^{3} \ldots d u^{1} \wedge d u^{4} \\
& 2
\end{aligned} \quad \text {. }
$$


Similarly we can assume $n_{\alpha \beta}$ is partially isotropic about some axis. Apart from L.R.S. solutions, we find only solutions in which $n_{\alpha \beta}$ is partially isotropic because some $n_{\gamma}$ are zero (types I, II; V, IV).

Now we can consider those cases in which the surfaces of transitivity are isotropic,

$$
R_{\alpha \beta}^{*}=\frac{1}{3} R^{*} \delta_{\alpha \beta}, \quad R^{*}=R_{a}^{* a},
$$

these surfaces then being three-surfaces of constant curvature $R^{*} / 6$.

Using a Fermi-propagated tetrad $\left(\Omega_{\beta}=0\right)$ in (3.7a), the isotropy of $R_{\alpha \beta}^{*}$ implies [3] [using definition (3.14)]

$$
\partial_{0} \sigma_{\alpha \beta}=-\theta \sigma_{\alpha \beta} \Leftrightarrow \sigma_{\alpha \beta}=\frac{\Sigma_{\alpha \beta}}{l^{3}}, \quad \Sigma_{\alpha \beta}^{\cdot}=0
$$

the shear tensor being thus determined by the trace-free constant tensor $\Sigma_{\alpha \beta}$. Defining $\Sigma^{2}=\frac{1}{2} \Sigma_{\alpha \beta} \Sigma^{\alpha \beta},(7.7)$ implies

so substituting in (3.9) gives

$$
\sigma^{2}=\Sigma^{2} l^{-6}
$$

$$
R^{*}=6 \mathrm{Kl}^{-2}, \quad K^{\cdot}=0,
$$

where the constant $K$ is the curvature of the three-space on which $l=1$. Use of (7.8) and (1.3) allows integration of (3.2), if $l \neq 0$, to give

$$
3\left(l^{*}\right)^{2}-\Sigma^{2} l^{-4}-\left(l^{2} \mu\right)-\Lambda l^{2}=10 E, \quad E \cdot=0
$$

a generalisation of the Friedmann equation; comparison with (3.8) shows that $E$ is related to the $K$ of (7.9) by $3 K=-10 E$. With suitable rescaling of $l, K$ can be set to \pm 1 if it is not zero.

If we assume the barotropic equation of state $p=(\gamma-1) \mu$ where $\gamma$ is a constant then (1.3) integrates to give

$$
\mu=M l^{-3 \gamma}, \quad M^{\cdot}=0, \quad M>0,
$$

where $\mu \geqq p \geqq 0 \Rightarrow 2 \geqq \gamma \geqq 1$. Then (7.11) determines the behaviour of the matter term in (7.10) which we can now in principle integrate for $l(t)$ with given values of $\Sigma, M, \gamma, \Lambda$ and $E$. Suitable values of $\gamma$ are $\gamma=2(\Leftrightarrow p=\mu$, "stiff" matter $), \gamma=4 / 3\left(\Leftrightarrow p=\frac{1}{3} \mu\right.$, disordered radiation) and $\gamma=1$ ( $\Leftrightarrow p=0$, dust). We can, if we wish, add several such terms to represent non-interacting components of matter.

Using the tetrad $(2.14)$ in $(3.7 \mathrm{~b})$ we find $R_{\alpha \beta}^{*}$ is isotropic if and only if

$$
\begin{aligned}
0 & =a\left(n_{2}-n_{3}\right)=\left(n_{1}+n_{2}-n_{3}\right)\left(n_{1}-n_{2}\right) \\
& =\left(n_{2}+n_{3}-n_{1}\right)\left(n_{2}-n_{3}\right)=\left(n_{3}+n_{1}-n_{2}\right)\left(n_{3}-n_{1}\right)
\end{aligned}
$$


so that at least two of the $n_{\beta}$ are equal. Renumbering to make these $n_{2}, n_{3}$ we find (7.12) is satisfied either by

or

$$
n_{1}=0, \quad n_{2}=n_{3}
$$

$$
n_{1}=n_{2}=n_{3}=: m \text {. }
$$

Now $n_{2}=n_{3} \neq 0$ implies $\theta_{2}=\theta_{3}$ and $\sigma_{23}=0$; this is an L.R.S. solution of type $\mathrm{VII}_{0}$ or $\mathrm{VII}_{h}$. These cases are also invariant under groups of type $I$ and $V$ respectively, by (7.1) to (7.3), and the latter case is Robertson-Walker, since $\sigma_{\alpha \beta}=0$. Hence there are always axes such that $(7.13 \mathrm{~b})$ holds and $R^{*}=\frac{3}{2} m^{2}-6 a^{2}$. If $m \neq 0$ we have a type IX group and the time derivative of $(7.13 \mathrm{~b})$ shows it is Robertson-Walker $\left(R^{*}>0\right)$. If $m=0$ we have spaces invariant under a group of type I (if $a=0$ and $\left.R^{*}=0\right)$ or type $V\left(a \neq 0, R^{*}<0\right)$. This proves the results concerning Robertson-Walker models [for which (7.6) and (7.7) always hold] quoted in Section 5 and earlier in this section. Apart from the Robertson-Walker cases of type IX, these spaces are special cases of those examined in Section 6.

Theorem 7.1. The only spacetimes satisfying (1.1), (1.2) and (1.4) in which the three-surfaces of transitivity are isotropic are those of types $I$ and $V$ (in which these surfaces are necessarily isotropic) and a special case of type IX; in the last case the isotropy of the surfaces implies their isotropic embedding. If in addition the spacetime is L.R.S., the type $I$ and $V$ cases also admit groups of types $V I I_{0}$ and $V I I_{h}$ respectively.

This result is rather surprising. It includes all Robertson-Walker models [by (3.7a)]. Since groups of type VIII are rather closely related to those of type IX (one can go from one to the other by a complex coordinate transformation), one might have expected that a RobertsonWalker model with $R^{*}<0$ would be invariant under a simply transitive type VIII group but the appropriate group is type $\mathrm{V}$ (or $\left.\mathrm{VII}_{0}\right)^{6}$.

In each case there are Fermi-propagated shear eigenvectors [Lemmas $4.1,5.3$ or directly from (7.7)] and we can define lengths $l_{\alpha}$ along the principal directions of shear by $l_{1}:=X, l_{2}:=Y, l_{3}:=Z$ in (4.9) or (6.7). Then $\dot{l}_{\alpha} / l_{\alpha}=\theta_{\alpha}$ (no sum) and $l^{3}=$ const $\times l_{1} l_{2} l_{3}$. We have thus diagonalised $\Sigma_{\alpha \beta}$. Maximising (e.g.) $\Sigma_{11}$ with $\Sigma_{\alpha \beta}$ trace-free and $\Sigma$ constant, we find we may write

$$
\Sigma_{\beta \beta}=\frac{2}{\sqrt{3}} \Sigma \sin \alpha_{\beta} ; \alpha_{\beta}:=\alpha \pm(\beta-1) \frac{2 \pi}{3},
$$

where $\alpha$ is a constant and there is no sum over $\beta$. Then the relation of $l, l_{\gamma}$ and $\theta_{\gamma}$ is

$$
i_{\gamma}\left|l_{\gamma}=\theta_{\gamma}=l \cdot\right| l+\frac{2}{\sqrt{3} l^{3}} \Sigma \sin \alpha \quad(\text { no sum })
$$


which can be integrated as

$$
l_{\gamma}(t)=l(t) \exp \left(\frac{2}{\sqrt{3}} \Sigma \sin \alpha_{\gamma} \int \frac{d t}{l^{3}}\right) .
$$

Using the coordinates (4.9) or (6.7) the $l_{\gamma}$ completely determine the behaviour of the models.

Theorem 7.2. In spaces satisfying the conditions of Theorem 7.1, the field equations reduce to $(7.10),(7.14),(7.16)$ where $\alpha, \Sigma, E$ are constants related by $E<0 \Rightarrow \Sigma=0 ; E>0 \Rightarrow \alpha=0$.

[The last restriction follows from (5.5).]

In each case we can specify the behaviour of the matter [e.g. by choosing $M, \gamma$ in (7.7)]; integrate (7.10); and then integrate (7.16) for allowed $\alpha, \Sigma$ and $E$. When $\Sigma=0$ this procedure gives the RobertsonWalker solutions explicitly.

For type IX groups we have only the Robertson-Walker case with $K=+1$. A special case is when $\theta=0$ and (7.10) is no longer a first integral of (3.2); we find $m$ is a positive constant, $\mu+p=\frac{1}{2} m^{2}$, $\Lambda=\frac{1}{2} \mu+\frac{3}{2} p$, and we have the Einstein static universe (which is invariant under a type IX group only) unless $\mu+p=0$ when we have flat empty space (since $\mu \geqq 0, p \geqq 0$ ) and the group degenerates to type I. If the space is empty the solution requires $A>0$ and is the de Sitter universe.

For type I groups we can integrate to obtain the well-known solutions for most reasonable behaviour of matter $([2,6,14,23-26])$. The solutions for "stiff" matter are

$$
\begin{array}{lll}
\Lambda>0, & l^{3}=c \sinh \sqrt{3 \Lambda} t, & l_{\beta}=l\left(\tanh \frac{\sqrt{3 \Lambda} t}{2}\right)^{n_{\beta}}, \\
\Lambda=0, & l^{3}=\sqrt{3\left(\Sigma^{2}+M\right) t,}, & l_{\beta}=l t^{n_{\beta}}, \\
\Lambda<0, & l^{3}=c \sin \sqrt{-3 \Lambda} t, & l_{\beta}=l\left(\tan \frac{\sqrt{-3 \Lambda} t}{2}\right)^{n_{\beta}}
\end{array}
$$

where

$$
c=\frac{\sqrt{\Sigma^{2}+M}}{\sqrt{|\bar{M}|}}, \quad n_{\beta}=\frac{2 \Sigma \sin \alpha_{\beta}}{3 \sqrt{\Sigma^{2}+M}} .
$$

If we set $M=0$ we derive the vacuum solutions (the $A=0$ case being Kasner's solution [28]). The "stiff" matter term and the shear terms in (7.10) have the same $l$-dependence, so the vacuum and stiff matter solutions are essentially the same.

For groups of type V, we obtain Heckmann and Schücking's representation $[6,10]$. The first integral Eq. (7.10) can be solved if $\Sigma=0$ (Robertson-Walker) for various behaviour of matter; for empty space (giving Joseph's solution [31] if $\Lambda=0$ ); for $\Lambda=0$ with $\gamma=2,5 / 3,4 / 3$, 
or 1 and $\Lambda \neq 0$ with $\gamma=2$ or $4 / 3$. All except isotropic models and Joseph's solution require elliptic integrals. As in type $\mathrm{I}$, the stiff matter and empty space solutions are essentially the same. In the type $V$ and IX cases it often helps to introduce a new time variable [e.g. by taking $t^{\prime}=\int d t / X$ which implies $\boldsymbol{e}_{0}=A\left(t^{\prime}\right) \partial / \partial t^{\prime}$ and gives the $\boldsymbol{e}_{0} / \boldsymbol{e}_{\mathbf{1}}$ surfaces in conformally flat coordinates].

Even when (7.10), (7.16) are not analytically integrable we may use (7.10) together with (7.15), (7.7) and (1.3) to give analytic expressions for the expansion $\theta$, principal expansions $\theta_{v}$, density of matter $\mu$ and shear $\sigma_{\alpha \beta}$ in terms of the average length $l$. This gives considerable quantitative knowledge, as we lack only the relation of $l$ to the proper time $t$.

In particular we see from (7.10) and (3.2) that if $\mu \geqq p \geqq 0$ the type IX solutions with $\Lambda=0$ always reach a point where $\theta=0$ and $\theta^{\circ}<0$. So the solution reconverges to a second singularity. In types I and $V$ the solution expands at all times.

\section{Vacuum Solutions and 0ther Special Cases}

We can obtain from our equations a number of explicit solutions for a vacuum $(\mu=p=0)$, sometimes requiring the extra condition $\Lambda=0$. Our classification is not dependent on $\mu \neq 0$ or $p \neq 0$, so we obtain all empty space solutions invariant under a group $G_{3}$ simply transitive on spacelike hypersurfaces, on choosing the normals to these hypersurfaces as the vector field $u^{a}$ (now not defined by $T_{a b} \cdot$ ).

First we consider all vacuum solutions in which $R_{\alpha \beta}^{*}$ is isotropic. For type $I$, these are the solutions (7.17) with $M=0$. The L.R.S. subclass $\left(\theta_{2}=\theta_{3}\right)$ is the case $A_{3}$ of EHLERs and KundT [35] (in different coordinates). For type IX there is only the de Sitter space $(\Lambda>0)$; and for type $V$ we have Joseph's solution [31] and its generalisation to nonzero $\Lambda$. The solutions with $R_{\alpha \beta}^{*}$ isotropic include the completely isotropic solutions $(\sigma=0)$, which are invariant under a group of motions $G_{10}$ and are the (conformally flat) four-spaces of constant curvature. If the curvature of the four-space is positive $(\Lambda>0)$ the group is type $I, V$ or $\mathrm{IX}$; if it is zero $(\Lambda=0)$ the group is type $\mathrm{I}$ or $\mathrm{V}$; and if it negative, the group is type $V^{8}$.

We can also obtain all vacuum L.R.S. solutions in our class of spaces ${ }^{9}$. The L.R.S. classification of Section 7 holds also in the vacuum case. The type I solutions are those mentioned above. For the groups of types II, IX and VIII [or III with $n_{\beta}^{\beta} \neq 0$, see (7.5)] we obtain the

${ }^{8}$ Or, in each case the appropriate related groups of type $\mathrm{VIII}_{0}$ or $\mathrm{VII}_{h}$ : see Theorem 7.1.

9 M. CAHEN and L. Defrise have found all vacuum L.R.S. solutions (Ref. [36] and private communication). 
NUT spaces [37] (the type IX case being Taub-NUT space [5, 37] investigated in detail by MrSNER and TAUB [38]). These may be integrated with non-zero $\Lambda^{10}$ giving in the coordinates of $(4.9)$

$$
\begin{aligned}
u & =\int X d t, \quad Y^{2}=u^{2}+\left(N_{1}\right)^{2}, \\
Y^{2} X^{2} & =k+m u-N_{1} N_{2} u^{2}+\frac{1}{2} \Lambda\left(N_{1}\right)^{2} u^{2}+\frac{1}{3} \Lambda u^{4},
\end{aligned}
$$

where $k=\frac{1}{4}\left(N_{1}\right)^{3} N_{2}-\frac{1}{16} \Lambda\left(N_{1}\right)^{4}$ and $m$ is an arbitrary constant $\left(N_{1} N_{2}\right.$ being the NUT parameter corresponding to $\mu_{0}$ in Ref. [37]). The type II case has $N_{2}=0$, types VIII and IX having $N_{2} \neq 0$ and opposite signs of $N_{1}$. The axis of symmetry is $\boldsymbol{e}_{1}$. If the group is Bianchi type III with $n_{\beta}^{\beta}=0$ we obtain the spatially homogeneous part of the "pseudoSchwarzschild" space (type $A_{2}$ in Ref. [35]) which is easily generalised to $\Lambda \neq 0$. The only empty space with homogeneous spatial sections and a one-parameter isotropy group of spatial rotations which is not included in those obtained above is the spatially homogeneous part of the Schwarzschild solution (type $A_{1}$ in Ref. [35]) which corresponds to the spaces of class I in KantowskI and SACHS $[32,33]$ and does not have a threeparameter group of motions simply transitive on the spacelike hypersurface of transitivity of the group $G_{4}$.

The type II and III $\left(n_{\beta}^{\beta} \neq 0\right)$ cases in the NUT spaces reduce to the Schwarzschildian spaces $\left(A_{2}\right.$ and $A_{3}$ of Ref. [35]) by smooth transitions to zero of the appropriate parameters. But there is no such smooth transition for the space of type IX (nor would there be in type VIII if we did not rewrite in terms of the group of type III).

We may also obtain some vacuum solutions with $n_{\beta}^{\beta}=0$. For types I and $\mathrm{V}$ these are as above. In type $\mathrm{VI}_{h}$ the solution with $\Lambda=0$ is [in the coordinates of $(6.7)]$

$$
\begin{aligned}
t & =\int X d u, \quad X^{2}=\left(\sinh 2 a_{0} u\right)^{1+k^{2}}\left(\tanh a_{0} u\right)^{k \sqrt{3+k^{2}}} \\
Y^{2} & =\left(\sinh 2 a_{0} u\right)^{1+k}\left(\tanh a_{0} u\right)^{\sqrt{3+k^{2}}} \\
Z^{2} & =\left(\sinh 2 a_{0} u\right)^{1-k}\left(\tanh a_{0} u\right)^{-\sqrt{3+k^{2}}}
\end{aligned}
$$

where $k=q_{0} / a_{0}$. This gives Joseph's (type V) solution when $k=0$. Expanding the solution (8.2) in powers of $u$ and then letting $\alpha_{0}$ go to zero gives the vacuum space of type $\mathrm{VI}_{0}$ which may also be derived by setting $M=0$ in (6.8). We have not found a general solution for $\mathrm{Bbii}$ with $n_{\beta}^{\beta}=0$ (or otherwise). However, the spatially homogeneous part of the special solution of CoLLINSON and French [40], with a Weyl tensor of Petrov type III is of Case Bbii.

${ }^{10}$ Using a method due to J. M. Stewart. These solutions are part of a general family found by B. CARTER [39]. 
Table 4. Possible group types which occur in special cases. "Integrable" means integrable at least for dust. " $\leftrightarrow$ " identifies the same model it it has more than one group of motions simply transitive on the $S_{3}$. " $a$ " means $n_{\beta \alpha}=0$; " $b$ " means $n_{\beta \alpha} \neq 0$. This table includes all cases so far analytically integrated

\begin{tabular}{|c|c|c|c|c|c|}
\hline & \multirow[b]{2}{*}{ Case } & \multicolumn{3}{|c|}{ special cases, not Robertsox-WaLKER } & \multirow{2}{*}{$\begin{array}{l}\text { Robertson- } \\
\text { Walker cases }\end{array}$} \\
\hline & & $\begin{array}{l}n_{\beta}^{\beta}=0 \\
\text { [coords (6.5), } \\
\text { field Eqs. } \\
(6.7) \text { ] }\end{array}$ & $\begin{array}{l}\text { isotropic } \\
3 \text {-spaces } \\
\text { [coords, and } \\
\text { field Eqs. } \\
(7.10)(7.16)]\end{array}$ & $\begin{array}{l}\text { L.R.S. } \\
\text { (coords. and } \\
\text { field Eqs. } \\
\text { see }[1,2] \text { ) }\end{array}$ & \\
\hline \multirow[t]{4}{*}{ A } & \multirow{2}{*}{$\begin{array}{l}\text { Aa: type I } \\
\text { (always inte- } \\
\text { grable) }\end{array}$} & \multirow[t]{2}{*}{ necessarily } & necessarily & 1 & $\mathrm{I}(K=0)$ \\
\hline & & & $\begin{array}{l}\text { (see Refs. } \\
{[23] \text { to }[26] \text { ) }}\end{array}$ & $\uparrow$ & $\uparrow$ \\
\hline & \multirow[t]{2}{*}{$\begin{array}{l}\text { Ab: [coords } \\
(4.5), \text { Eqs. } \\
(4.9)]\end{array}$} & \multirow[t]{2}{*}{$\mathrm{VI}_{0}$} & $\mathrm{VII}_{0}$ (L.R.S.) & $\begin{array}{l}\text { VII }_{0} \\
\text { II, IX }\end{array}\left(\begin{array}{l}\text { see Ref. } \\
[5,38])\end{array}\right)$ & \multirow[t]{2}{*}{$\begin{array}{l}\operatorname{VII}_{0}(K=0) \\
\mathrm{IX}(K=+1)\end{array}$} \\
\hline & & & & $\rightarrow \mathrm{VIII}$ & \\
\hline \multirow[t]{3}{*}{ B } & $\begin{array}{l}\text { Ba: V [Eqs. } \\
\text { (6.7), inte- } \\
\text { grable] }\end{array}$ & necessarily & $\begin{array}{l}\text { necessarily } \\
\text { (Refs. [31, 6]) }\end{array}$ & none & $V(K=-1)$ \\
\hline & Bbi & $\mathrm{VI}_{h}, \mathrm{III}$ & none & $\begin{aligned} \rightarrow & \operatorname{III}\left(n_{\beta_{\beta}}^{\beta} \neq 0\right) \\
& \operatorname{III~}\left(n_{\beta}^{\beta}=0\right) \\
& (\operatorname{Ref} .[32])\end{aligned}$ & $\stackrel{\downarrow}{\mathrm{VII}_{h}}(K=-1)$ \\
\hline & $\begin{array}{l}\text { Bbii: } \mathrm{VI}_{h}, \\
h=-1 / 9\end{array}$ & $\mathrm{VI}_{h}$ & none & none & none \\
\hline
\end{tabular}

Finally we have the general solution for type II with $\Lambda=0$ given by TAUB [5]. It seems probable that further investigation of the field equations would yield further analytic spatially homogeneous vacuum solutions.

There are a number of analytic solutions in which one of the $\theta_{\nu}=0$; none of these are physically interesting in that they all have zero or negative redshift in some direction and may involve negative pressures or densities. We shall therefore not give them here.

To conclude our survey of special cases of spatially homogeneous solutions with hypersurfaces orthogonal to the fluid flow vector we have summarised our results for the main classes of such solutions in Table 4.

\section{Conclusion}

We have examined in some detail the properties of the solutions of Einstein's field equations for a perfect fluid which have homogeneous 
spatial sections orthogonal to the fluid flow, in the sense of admitting a three-parameter group of motions simply transitive on these surfaces. This has been done using an orthonormal tetrad technique.

The models fall into two broad classes distinguished by whether the vector $a^{\beta}$ (defined in Section 2) is or is not zero; that is, whether the three-vector $C_{\beta \alpha}^{\alpha}$ (formed from the group structure constants) vanishes or not. We were able to find a canonical form for the tetrad-coordinate relation whenever $C_{\beta \alpha}^{\alpha}=0$ (Class A) and to use this to describe many properties of these solutions. Without explicitly finding coordinates in Class $\mathrm{B}\left(C_{\alpha \beta}^{\beta} \neq 0\right)$ we were able to describe general properties of these solutions (see Sections 4 and 5).

We further investigated a number of special subclasses. These included three subsets of our models with especially simple features, which were i) solutions in which $n_{\beta}^{\beta}=0\left(n_{\alpha \beta}\right.$ being defined in Section 2$)$, ii) solutions with local rotational symmetry (i.e. a group of motions multiply transitive on the homogeneous spatial sections), iii) solutions in which the surfaces of homogeneity are isotropic three-spaces. In all these cases canonical forms of the tetrad-coordinate relations were found, and in most cases the field equations were partially integrated.

The only fluid cases which have been analytically integrated for a wide range of barotropic fluids are those in which the group of motions is of Bianchi type I or III with $n_{\beta}^{\beta}=0$. These fall into the special subsets detailed above. The known spatially homogeneous vacuum solutions are discussed in Section 8.

When one cannot integrate analytically, one may either integrate numerically [12-14] or use approximation techniques $[11,15,24,25,30]$ to determine properties of the models. In particular one can investigate the behaviour when nearly isotropic and when near the singularity. These cases may respectively provide reasonable approximations for the present-day universe, and for the early behaviour of the universe, the latter determining the primeval element formation.

In a subsequent paper we will discuss some of these questions. This will also contain a detailed discussion of the properties of observations in these cosmological models. The most interesting of these properties is the existence of discrete isotropies.

Acknowledgements. We would like to thank Drs. C. G. BeHr, S. W. Hawking and B. Sсниidt for many helpful discussions, and Dr. L. Shepley for useful comments at an early stage in the work. One of us (G.F.R.E.) would like to thank the members of the Hamburg Relativity Seminar for their generous hospitality, and especially Dr. W. KUNDT for fruitful discussions which helped materially in the work of this paper. 


\section{Appendix I}

With the specialisation (2.14) the equations read as follows

$$
\begin{aligned}
& \partial_{0} a+\theta_{1} a=0, \\
& a\left(\Omega_{3}-\sigma_{12}\right)=0 \text {, } \\
& \alpha\left(\Omega_{2}+\sigma_{13}\right)=0 \text {; } \\
& \partial_{0} n_{1}+\left(\theta_{2}+\theta_{3}-\theta_{1}\right) n_{1}=0, \\
& \left(n_{1}+n_{2}\right) \sigma_{12}+\left(n_{1}-n_{2}\right) \Omega_{3}=0, \\
& \left(n_{3}+n_{1}\right) \sigma_{13}+\left(n_{3}-n_{1}\right) \Omega_{2}=0, \\
& \partial_{0} n_{2}+\left(\theta_{1}+\theta_{3}-\theta_{2}\right) n_{2}=0, \\
& \left(n_{2}+n_{3}\right) \sigma_{23}+\left(n_{2}-n_{3}\right) \Omega_{1}=0 \text {, } \\
& \partial_{0} n_{3}+\left(\theta_{1}+\theta_{2}-\theta_{3}\right) n_{3}=0 \text {; } \\
& \Lambda=\partial_{0} \theta+\theta_{1}^{2}+\theta_{2}^{2}+\theta_{3}^{2}+2 \sigma_{23}^{2}+2 \sigma_{13}^{2}+2 \sigma_{12}^{2} \\
& +\frac{1}{2} \mu+\frac{3}{2} p \text {; } \\
& a\left(2 \theta_{1}-\theta_{2}-\theta_{3}\right)+\sigma_{23}\left(n_{2}-n_{3}\right)=0, \\
& 3 a \sigma_{12}+\sigma_{13}\left(n_{3}-n_{1}\right)=0, \\
& 3 a \sigma_{13}+\sigma_{12}\left(n_{1}-n_{2}\right)=0 \text {; } \\
& \Lambda+\frac{1}{2}(\mu-p)=\partial_{0} \theta_{1}+\theta \theta_{1}-2 a^{2}+\frac{1}{2} n_{1}^{2} \\
& -\frac{1}{2}\left(n_{2}-n_{3}\right)^{2}+2 \sigma_{12} \Omega_{3}-2 \sigma_{13} \Omega_{2}, \\
& =\partial_{0} \theta_{2}+\theta \theta_{2}-2 a^{2}+\frac{1}{2} n_{2}^{2} \\
& -\frac{1}{2}\left(n_{1}-n_{3}\right)^{2}+2 \sigma_{23} \Omega_{1}-2 \sigma_{12} \Omega_{3}, \\
& =\partial_{0} \theta_{3}+\theta \theta_{3}-2 a^{2}+\frac{1}{2} n_{3}^{2} \\
& -\frac{1}{2}\left(n_{1}-n_{2}\right)^{2}+2 \sigma_{13} \Omega_{2}-2 \sigma_{23} \Omega_{1}, \\
& 0=\partial_{0} \theta_{12}+\theta \sigma_{12}+\left(\theta_{2}-\theta_{1}\right) \Omega_{3} \\
& +\sigma_{13} \Omega_{1}-\sigma_{23} \Omega_{2} \\
& 0=\partial_{0} \sigma_{13}+\theta \sigma_{13}+\left(\theta_{1}-\theta_{3}\right) \Omega_{2} \\
& +\sigma_{23} \Omega_{3}-\sigma_{12} \Omega_{1} \\
& 0=\partial_{0} \sigma_{23}+\theta \sigma_{23}+\left(\theta_{3}-\theta_{2}\right) \Omega_{1} \\
& +\sigma_{12} \Omega_{2}-\sigma_{13} \Omega_{3}+a\left(n_{2}-n_{3}\right) \text {; }
\end{aligned}
$$




$$
\begin{aligned}
& \theta_{1} \theta_{2}+\theta_{2} \theta_{3}+\theta_{3} \theta_{1} \\
& =\mu+\Lambda+3 a^{2}+\frac{1}{4}\left(n_{1}^{2}+n_{2}^{2}+n_{3}^{2}-2 n_{2} n_{3}-2 n_{1} n_{3}-2 n_{1} n_{2}\right) \\
& +\sigma_{12}^{2}+\sigma_{13}^{2}+\sigma_{23}^{2} \\
& R_{12}^{*}=R_{13}^{*}=0, \quad R_{23}^{*}=a\left(n_{2}-n_{3}\right), \\
& R_{11}^{*}=-2 a^{2}+\frac{1}{2} n_{1}^{2}-\frac{1}{2}\left(n_{2}-n_{3}\right)^{2}, \\
& R_{22}^{*}=-2 a^{2}+\frac{1}{2} n_{2}^{2}-\frac{1}{2}\left(n_{3}-n_{1}\right)^{2}, \\
& R_{33}^{*}=-2 a^{2}+\frac{1}{2} n_{3}^{2}-\frac{1}{2}\left(n_{1}-n_{2}\right)^{2} ; \\
& R^{*}=-6 a^{2}+\frac{1}{2}\left(2 n_{1} n_{2}+2 n_{1} n_{3}+2 n_{2} n_{3}-n_{1}^{2}-n_{2}^{2}-n_{3}^{2}\right) ; \\
& H_{11}=-\frac{3}{2} n_{1} \sigma_{11}+\frac{1}{2}\left(n_{2}-n_{3}\right)\left(\theta_{2}-\theta_{3}\right) \text {, } \\
& H_{22}=-\frac{3}{2} n_{2} \sigma_{22}+\frac{1}{2}\left(n_{3}-n_{1}\right)\left(\theta_{3}-\theta_{1}\right) \text {, } \\
& H_{33}=-\frac{3}{2} n_{3} \sigma_{33}+\frac{1}{2}\left(n_{1}-n_{2}\right)\left(\theta_{1}-\theta_{2}\right), \\
& H_{12}=\frac{1}{2}\left(n_{3}-2 n_{1}-2 n_{2}\right) \sigma_{12}+\frac{1}{2} a \sigma_{13} \text {, } \\
& H_{13}=\frac{1}{2}\left(n_{3}-2 n_{1}-2 n_{3}\right) \sigma_{13}-\frac{1}{2} a \sigma_{12} \text { ， } \\
& H_{23}=\frac{1}{2}\left(n_{1}-2 n_{3}-2 n_{2}\right) \sigma_{23}+\frac{1}{2} a\left(\theta_{3}-\theta_{2}\right), \\
& E_{12}=\sigma_{13} \sigma_{23}-\theta_{3} \sigma_{12} \text {, } \\
& E_{23}=\sigma_{12} \sigma_{13}-\theta_{1} \sigma_{23}-a\left(n_{2}-n_{3}\right) \text {, } \\
& E_{13}=\sigma_{12} \sigma_{23}-\theta_{2} \sigma_{13} \text {, } \\
& E_{11}=\frac{2}{3}(\mu+\Lambda)+2 a^{2}-\frac{1}{2} n_{1}^{2}+\frac{1}{2}\left(n_{2}-n_{3}\right)^{2} \\
& -\theta \theta_{1}+\theta_{1}^{2}+\sigma_{12}^{2}+\sigma_{13}^{2} \\
& E_{22}=\frac{2}{3}(\mu+\Lambda)+2 a^{2}-\frac{1}{2} n_{2}^{2}+\frac{1}{2}\left(n_{3}-n_{1}\right)^{2} \\
& -\theta \theta_{2}+\theta_{2}^{2}+\sigma_{23}^{2}+\sigma_{12}^{2} \text {, } \\
& E_{33}=\frac{2}{3}(\mu+\Lambda)+2 \alpha^{2}-\frac{1}{2} n_{3}^{2}+\frac{1}{2}\left(n_{1}-n_{2}\right)^{2} \\
& -\theta \theta_{3}+\theta_{3}^{2}+\sigma_{13}^{2}+\sigma_{23}^{2} \text {. }
\end{aligned}
$$




\section{Appendix II}

With the specialisation (2.19) the equations are

$$
\begin{gathered}
\partial_{0} a+\theta_{1} a=0, \\
a\left(\Omega_{3}-\sigma_{12}\right)=0, \\
a\left(\Omega_{2}+\sigma_{13}\right)=0 ; \\
r\left(\Omega_{3}-\sigma_{12}-\Omega_{2}-\sigma_{13}\right)=0, \\
\partial_{0} r+\theta_{2} r-r\left(\sigma_{23}+\Omega_{1}\right)+q\left(\Omega_{3}-\sigma_{12}\right)=0, \\
\partial_{0} r+\theta_{3} r-r\left(\sigma_{23}-\Omega_{1}\right)-q\left(\sigma_{13}+\Omega_{2}\right)=0, \\
r\left(\Omega_{3}+\sigma_{12}\right)+q\left(\sigma_{23}-\Omega_{1}\right)=0, \\
\partial_{0} q+\theta_{1} q-2 r\left(\sigma_{12}+\sigma_{13}\right)=0, \\
r\left(\sigma_{13}-\Omega_{2}\right)+q\left(\sigma_{23}+\Omega_{1}\right)=0 ; \\
\Lambda=\partial_{0} \theta+\theta_{1}^{2}+\theta_{2}^{2}+\theta_{3}^{2}+2 \sigma_{12}^{2}+2 \sigma_{13}^{2}+2 \sigma_{23}^{2} \\
+\frac{1}{2} \mu+\frac{3}{2} p ;
\end{gathered}
$$

$$
\begin{aligned}
a\left(2 \theta_{1}-\theta_{2}-\theta_{3}\right)+q\left(\theta_{3}-\theta_{2}\right)+r\left(\sigma_{13}-\sigma_{12}\right) & =0, \\
\sigma_{12}(q+3 a)+r\left(\theta_{1}-\theta_{3}-\theta_{23}\right) & =0, \\
\sigma_{13}(q-3 a)+r\left(\theta_{1}-\theta_{2}-\sigma_{23}\right) & =0 ;
\end{aligned}
$$

$$
\begin{aligned}
\Lambda+\frac{1}{2}(\mu-p)=\partial_{0} \theta_{1} & +\theta \theta_{1}-2 a^{2}-2 q^{2} \\
& +2 \Omega_{3} \sigma_{12}-2 \sigma_{13} \Omega_{2} \\
=\partial_{0} \theta_{1} & +\theta \theta_{2}-2 a^{2}-2 r^{2}-2 q a \\
& +2 \Omega_{1} \sigma_{23}-2 \sigma_{12} \Omega_{3} \\
=\partial_{0} \theta_{3} & +\theta \theta_{3}-2 a^{2}-2 r^{2}+2 a q \\
& +2 \Omega_{2} \sigma_{13}-2 \sigma_{23} \Omega_{1},
\end{aligned}
$$

$$
\begin{array}{r}
\partial_{0} \sigma_{12}+\theta \sigma_{12}+\left(\theta_{2}-\theta_{1}\right) \Omega_{3}+\sigma_{13} \Omega_{1}-\sigma_{23} \Omega_{2}+2 r q=0,(12) \\
\partial_{0} \sigma_{13}+\theta \sigma_{13}+\left(\theta_{1}-\theta_{3}\right) \Omega_{2}+\sigma_{23} \Omega_{3}-\sigma_{12} \Omega_{1}+2 q r=0,(13) \\
\partial_{0} \theta_{23}+\theta \sigma_{23}+\left(\theta_{3}-\theta_{2}\right) \Omega_{1}+\sigma_{12} \Omega_{2}-\sigma_{13} \Omega_{3}+2 r^{2}=0 ;(23) \\
\Lambda+\mu+3 a^{2}+q^{2}+\sigma_{12}^{2}+\sigma_{13}^{2}+\sigma_{23}^{2}=\theta_{1} \theta_{2}+\theta_{2} \theta_{3}+\theta_{1} \theta_{3} .
\end{array}
$$




\section{References}

1. Eluis, G. F. R.: J. Math. Phys. 8, 1171 (1967).

2. Stewart, J. M., and G. F. R. Ellis: J. Math. Phys. 9, 1072 (1968).

3. Ehlers, J.: Akad. Wiss. Lit. (Mainz), Abhandl. Math.-Nat. Kl. No. 11 (1961).

4. Eisenhart, L. P.: Continuous groups of transformations. New York: Dover 1961.

5. Taub, A. H.: Ann. Math. 53, 472 (1951).

6. Heckmann, O., and E. Schücking: Solvay Conference Report. Brussels: Edition Stoops 1958; and Chap. 11 of Gravitation: An introduction to current research, ed. L. WitTen, New York: Wiley 1962.

7. Gödel, K.: Proc. Intern. Congr. Math., Cambridge, Mass. 1, 175 (1952).

8. Branchi, L.: Lezioni sulla teoria dei gruppi continui finiti transformazioni. Pisa: Spoerri 1918.

9. Ellis, G. F. R.: Ph. D. Thesis, Cambridge University (1964).

10. Estabrook, F. B., H. D. Wahlquist, and C. G. Behr: J. Math. Phys. 9, 497 (1968).

11. Misner, C. W.: Astrophys. J. 151, 431 (1968).

12. BEHR, C. G.: Z. Astrophys. 54, 268 (1962), and Astron. Abhandl. Hamburg Sternwarte VII, 5 (1965).

13. Sheplex, L.: Proc. Natl. Acad. Sci. U.S. 52, 1403 (1964) and Ph. D. Thesis, Princeton University (1967).

14. Saunders, P. T.: Ph. D. Thesis, King's College, London University (1967).

15. Hawking, S. W.: Monthly Notices Poy. Astron. Soc. 142, 129 (1969).

16. 一, and G. F. R. Elurs: Phys. Letters 17, 246 (1965).

17. Raychaudhuri, A.: Phys. Rev. 98, 1123 (1955).

18. Estabrook, F. B., and H. D. Wahlquist: J. Math. Phys. 5, 1629 (1964); 7, 894 (1966), and JPL Tech. Rpt 32-868, Caltech. (1966).

19. SchückIng, E.: Hamburg Relativity Seminar (1962); W. Kundt: Hamburg Relativity Seminar (1963); C. G. BeHR, unpublished.

20. Jordan, P., J. Ehlers, and W. Kundt: Akad. Wiss. Lit. (Mainz), Abhandl. Math.-Nat. Kl. No. 7 (1960).

21. Sandage, A.: J. Soc. Ind. Appl. Math. 10, 781 (1962).

22. Petrov, A. Z.: Einstein-Räume (transl. H.-J. Treder). Berlin: Akad. Verlag 1964.

23. Raychaudhuri, A.: Proc. Phys. Soc. (London) 73, 263 (1958).

24. Robinson, B. B.: Proc. Natl. Acad. Sci. U.S. 47, 1852 (1961).

25. Thorne, K. S.: Astrophys. J. 148, 51 (1967).

26. Doroshkevich, A. S.: Astrophysics 1, 138 (1965).

27. JACOBS, K. C.: Astrophys. J. 153, 661 (1968).

28. Kasner, E.: Am. J. Math. 43, 217 (1921).

29. Dautcourt, G., A. Papapetrou and H. Treder: Ann. Physik 9, 330 (1962)

30. Misner, C. W.: Preprint, University of Maryland (1968).

31. Joseph, V.: Proc. Cambridge Phil. Soc. 62, 87 (1966).

32. Kantowski, R., and R. K. SAchs: J. Math. Phys. 7, 443 (1966).

33. - Ph. D. Thesis, University of Texas (1966).

34. Grishchuk, L. P.: Soviet Astron.-A. J. 11, 881 (1968); Russian orig., Astron. Zh. 44, 1097 (1967).

35. Ehlers, J., and W. KundT: Chap. 2 of Gravitation: An introduction to current research, ed. L. Witrten. New York: Wiley 1962.

36. Defrise, L.: Acad. Roy. Belg., Bull. classe Sci. 53, 827 and 1276 (1967).

37. Newman, E. T., T. Unti, and L. A. Tamburino: J. Math. Phys. 4, 915 (1963). 
38. Misner, C. W.: J. Math. Phys. 4, 924 (1963); and in: Relativity theory and astrophysics. Volume I, ed. J. EHLERs, Rhode Island, A.M.S. (1967). Misner, C. W., and A. H. TAub: J.E.T.P. 55, 233 (1968) in Russian.

39. Carter, B.: Phys. Letters 26 A, 399 (1968).

40. Collinson, C. D., and D. C. French: J. Math. Phys. 8, 701 (1967).

\author{
G. F. R. Eluis \\ M. A. H. MaCCALLUM \\ Department of Applied Mathematics \\ and Theoretical Physics \\ Silver Street, Cambridge, Great Britain
}

